\title{
Onkologie und Ökonomie - wie frei sind wir wirklich in der Behandlung von Patient*innen?
}

\author{
Bernhard Wörmann $^{a}$ Josef Hecken ${ }^{b}$ Han Steutel ${ }^{c}$ Ingo Tamm ${ }^{d}$ \\ Andrew Ullmann ${ }^{\mathrm{e}}$ Kirsten Kappert-Gonther ${ }^{f}$ Hermann Einsele ${ }^{g}$ Martin Siess $^{\mathrm{h}}$ \\ Christian Wylegalla \\ ${ }^{a}$ Medizinische Klinik mit Schwerpunkt Hämatologie, Onkologie und Tumorimmunologie, Campus Virchow- \\ Klinkum (CVK), Berlin, Deutschland; ${ }^{b}$ Gemeinsamer Bundesausschuss, Berlin, Deutschland; ${ }^{c}$ Verband Forschender \\ Arzneimittelhersteller e.V., Berlin, Deutschland; ${ }^{d}$ Onkologische Schwerpunktpraxis, Berlin, Deutschland; ${ }^{e}$ Obmann \\ Fraktion der Freien Demokraten im Deutschen Bundestag, Abgeordnetenbüro Deutscher Bundestag, Berlin, \\ Deutschland; ${ }^{\mathrm{f} B u ̈ n d n i s ~ 90 / D i e ~ G r u ̈ n e n, ~ B e r l i n, ~ D e u t s c h l a n d ; ~} 9$ Medizinische Klinik und Poliklinik II, Zentrum \\ Innere Medizin (ZIM), Universitätsklinikum Würzburg, Würzburg, Deutschland; ${ }^{h}$ Ärztlicher Direktor (Vorsitz), \\ Klinikum rechts der Isar der Technischen Universität München, München, Deutschland; 'Pflegedienstleitung \\ Universitätsklinikum Freiburg, Freiburg, Deutschland
}

\section{Arzneimittelpreise Nutzenbewertung und mehr? Referent: Bernhard Wörmann, Berlin}

In Deutschland werden seit dem 1. Januar 2011 alle neu zugelassenen, verschreibungspflichtigen Arzneimittel der frühen Nutzenbewertung unterzogen [1]. Das Instrument, das nach dem Gesetz zur Neuordnung des Arzneimittelmarktes (AMNOG) von 2010 (\$35a SGB V) zur Schaffung einer Preisverhandlungsbasis zwischen Kostenträgern und pharmazeutischen Unternehmen eingeführt wurde, hat auch international als etabliertes und funktionierendes Verfahren viel Anerkennung erfahren. Das strukturierte, stark formalisierte Verfahren wird mit hoher administrativer Effektivität durchgeführt (Abb. 1) [2]. Nahezu alle Nutzenbewertungen erfolgen fristgerecht, d.h. das AMNOG-Verfahren wird innerhalb der ersten 12 Monate nach Markteintritt eines Arzneimittels abgeschlossen. Gleichzeitig schafft das Gesetz einen Rahmen für die pharmazeutischen Unternehmen zur Einführung neuer Arzneimittel auf dem deutschen Markt. Im europäischen Vergleich gehört Deutschland derzeit zu den Ländern mit der frühesten Markteinführung neuer Arzneimittel.

\section{Innovationstreiber Onkologie}

Wie ein Blick auf die relative Verteilung der Fachgebiete bei der Entwicklung und Markteinführung neuer Arzneimittel zeigt, ist die Onkologie das Fachgebiet mit den meisten neu zugelassenen Arzneimitteln und Indikationen. Die Zahl der Verfahren hat insbesondere in der Onkologie weiter zugenommen, sodass die Statistik der abgeschlossenen Verfahren der frühen Nutzenbewertung nach Fachgebieten von den Onkologika angeführt wird [3] (Abb. 2). Dabei hat auch die Vielfalt der Indikationen zugenommen, wobei viele der innovativen Neuzugänge der letzten Jahre, darunter die Immuncheckpoint-Inhibitoren, aber auch zielgerichtete Arzneimittel wie Tyrosinkinase-Inhibitoren, indikationsübergreifend bei mehreren verschiedenen Tumorentitäten wirksam sind [4].

Trotz der enormen Verbreiterung des krebstherapeutischen Armamentariums und der wachsenden Behandlungsmöglichkeiten selbst in fortgeschrittenen Krankheitsstadien bleibt der klinische Forschungsbedarf unverändert hoch. So zählen onkologische Erkrankungen nach wie vor zu den Haupttodesursachen weltweit. In Deutschland erreichen immer noch $35-40 \%$ nicht das relative 5-Jahres-Überleben bzw. 39-46\% nicht das 10-Jahres- 


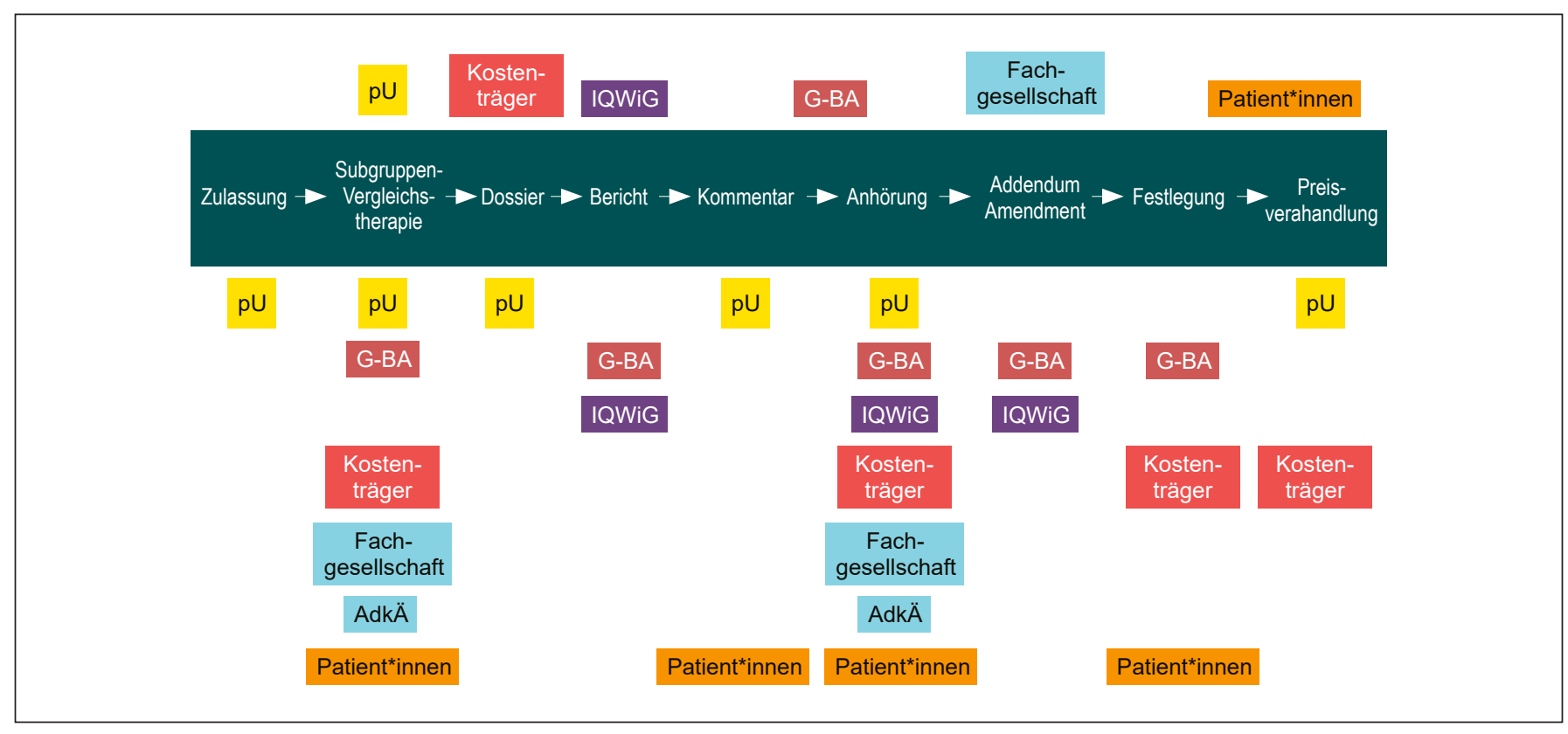

Abb. 1. Schematische Darstellung der frühen Nutzenbewertung von Arzneimitteln; mod. nach [2]. AkdÄ, Arzneimittelkommission der deutschen Ärzteschaft; G-BA, Gemeinsamer Bundesausschuss; IQWiG, Institut für Qualität und Wirtschaftlichkeit im Gesundheitswesen; pU, pharmazeutisches Unternehmen.

Abb. 2. AMNOG-Verfahren nach Fachgebieten: 2012-2020.

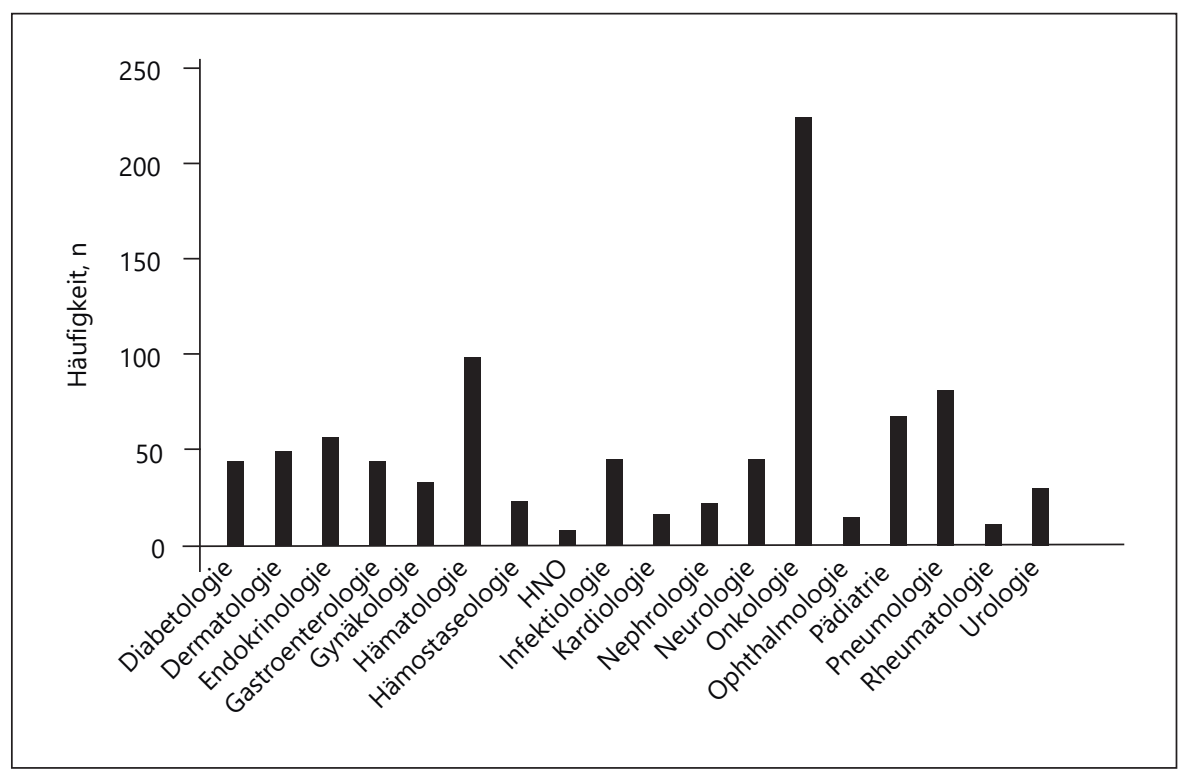

Überleben, wobei die Ergebnisse häufig in hohem Maße von der Tumorart und Verfügbarkeit tumorspezifischer Therapieoptionen abhängig sind [5].

$\mathrm{Zu}$ den Besonderheiten im frühen Nutzenbewertungsverfahren nach dem AMNOG gehört die vorgesehene Analyse von Subgruppen: Der Zusatznutzen wird innerhalb einer Indikation für definierte Subgruppen festgelegt. Im Prinzip können Subgruppenanalysen dazu dienen, patientenbedingte und therapiespezifische Variablen mit relevantem Einfluss auf den Einsatz eines Arzneimittels zu identifizieren - sei es im Sinne der präferenziellen
Gabe oder der Vermeidung [6]. Im Rahmen der Nutzenbewertung betrifft die Subgruppenbildung die Festlegung der zweckmäßigen Vergleichstherapie. Damit hat sie maßgeblichen Einfluss auf die endgültige Festlegung des Zusatznutzens. Das AMNOG-Verfahren sieht eine Nutzenbewertung aufgrund von Nachweisen des pharmazeutischen Unternehmens vor, die insbesondere die «Anzahl der Patient ${ }^{*}$ innen und Patient ${ }^{*}$ innengruppen» enthalten müssen, «für die ein therapeutisch bedeutsamer Zusatznutzen besteht.» Der Gemeinsame Bundesausschuss (GBA) setzt diese Vorgabe durch die Festlegung von Sub- 
gruppen zu Beginn eines neuen Verfahrens um [7]. Wird zum Beispiel eine zweckmäßige Vergleichstherapie festgelegt, für die es bekanntermaßen keine randomisierte Studie gibt, ist das Ergebnis «Zusatznutzen nicht belegt» vorhersehbar [6].

Zwischen 2011 und 2020 wurden nach der Systematik der frühen Nutzenbewertung in insgesamt 517 Verfahren 965 Subgruppen definiert und mit einer Festlegung abgeschlossen. Mit 59,6\% war das Ergebnis «Zusatznutzen nicht belegt» am häufigsten, mit deutlichem Abstand gefolgt von «Zusatznutzen gering» $(14,2 \%)$ und «Zusatznutzen nicht quantifizierbar» (13,4\%). Zwölf Prozent der neuen Arzneimittel wurde ein «beträchtlicher Zusatznutzen», 0,5\% ein «geringerer Zusatznutzen» und 0,3\% ein «erheblicher Zusatznutzen» zugesprochen. Das Fachgebiet der Onkologie schnitt im vergangenen Jahr im Vergleich zur Gesamtheit aller Verfahren besser ab: Der Anteil der Verfahren mit Zusatznutzen «nicht belegt» betrug in 2020 knapp 50\%, gefolgt von Zusatznutzen «beträchtlich» $(18,4 \%)$, «nicht quantifizierbar» $(17,6 \%)$, «gering» $(13,2 \%)$, «geringer» $(1,1 \%)$ und «erheblich» $(0,3 \%)$.

\section{Fachgesellschaften werden früher eingebunden}

Das Verfahren der frühen Nutzenbewertung auf der Basis der vorhandenen Evidenz wird von den wissenschaftlich-medizinischen Fachgesellschaften maßgeblich unterstützt: Von Anfang an haben sich die Fachgesellschaften durch Stellungnahmen und die Teilnahme an Anhörungen intensiv mit eingebracht [3]. In der Vergangenheit wurde immer wieder neben der Subgruppenbildung die Bedeutung einer zeitgerechten Einbindung der zuständigen Fachgesellschaften diskutiert: Um eine dem aktuellen Stand des Wissens entsprechende, wissenschaftlich abgesicherte Subgruppenbildung sowie Festlegung der zweckmäßigen Vergleichstherapie zu erreichen, hatte die Arbeitsgemeinschaft der Wissenschaftlichen Medizinischen Fachgesellschaften e.V. (AWMF) wiederholt die umfassende und rechtzeitige Einbeziehung gefordert [8]. Nach einem Beschluss des G-BA vom 20. Februar 2020 werden die Fachgesellschaften und die Arzneimittelkommission der deutschen Ärzteschaft (AkdÄ) nunmehr schon im Vorfeld der frühen Nutzenbewertung zu Fragen einer zweckmäßigen Vergleichstherapie hinzugezogen (Abb. 1): sowohl während einer Beratung zum Zeitpunkt der Studienplanung als auch zu anderen Zeitpunkten während der Nutzenbewertung [9]. Infolgedessen besteht die Hoffnung, dass sich nicht nur die Transparenz des Verfahrens weiter erhöhen, sondern auch die sehr hohe Anzahl von Amendments und Addenda im Verfahrensverlauf reduzieren lässt. Die frühzeitige Einbindung der Fachgesellschaften erwies sich bereits als praktikabel: Bis zum 9. Februar 2021 konnten bis auf eine Ausnahme sämtliche Anfragen (100/101), die im Bereich der Onkologie an die Fachgesellschaften gerichtet wurden, mit einer Stellungnahme beantwortet werden.

\section{Zugewinn an Information und Transparenz}

Das System der frühen Nutzenbewertung dient nicht nur dem raschen Marktzugang zu innovativen Medikamenten und der Wirtschaftlichkeit im Gesundheitswesen: Für die Fachgesellschaften liefert sie darüber hinaus wichtige Zusatzinformationen zum Schaden-NutzenVerhältnis eines neuen Arzneimittels [4]. Zudem sind zum Zeitpunkt der Nutzenbewertung viele Ergebnisse aus Zulassungsstudien noch nicht publiziert. Da das AMNOG-Verfahren aus Gründen der Informationstransparenz auch den Zugang zu bislang noch unveröffentlichten Daten einfordern kann, bietet das Nutzenbewertungsprozedere auch einen wertvollen Informationszugewinn über die publizierte Datenlage hinaus. Weiterhin zeigen die Erfahrungen mit einer späteren Nutzenbewertung, dass die Neubewertung auf der Basis reiferer Daten - wie z.B. ein Überlebensvorteil, der sich gegenüber der Vergleichstherapie erst bei der späteren Auswertung einer Studie mit längerer Nachbeobachtungsdauer abzeichnet - zu einem Ergebnis führen kann, das von der Erstbewertung abweicht.

In den Vorgaben der Arzneimittel-Nutzenbewertungsverordnung (AM-NutzenV; $\$ 2$ Abs. 3) von Anfang an berücksichtigt, gewinnen auch patient*innenrelevante Zielgrößen wie Morbidität und Erhalt von Lebensqualität neben den harten Endpunktparametern Todeszeitpunkt bzw. Mortalität zunehmend an Bedeutung. Der Anteil der Verfahren mit standardisierter Erfassung der Lebensqualität beläuft sich in der Onkologie auf mittlerweile über $80 \%$. Allerdings ist der Nutzen der erhobenen Daten zur Lebensqualität derzeit noch limitiert, da sie oftmals nicht engmaschig genug erfasst sind und über den Progress hinausgehen.

In Anbetracht der zunehmenden Komplexität der Behandlungsalgorithmen und der beständigen Neuzugänge auf dem onkologischen Arzneimittelmarkt bieten die evidenzbasierten Onkopedia-Leitlinien eine kompakte und leicht zugängliche Orientierungshilfe, die laufend aktualisiert und erweitert wird. Seit 2020 werden dort die Algorithmen zudem über rote Dreieckssymbole mit weiterführenden Informationen $\mathrm{zu}$ Wechselwirkungen, Nebenwirkungen und Leitlinientext sowie relevanten Studiendaten verlinkt (Abb. 3). Anhand von «Fact Sheets» können sich Behandler*innen auf einen Blick über die wichtigsten Outcomes der zugrunde liegenden Studien, deren Evidenzniveaus, den klinischen Nutzen («clinical benefit») sowie den vom G-BA festgelegten Zusatznutzen («additional benefits») des Onkologikums informieren (https://www.onkopedia.com/de/drug-assessment/guidelines). 


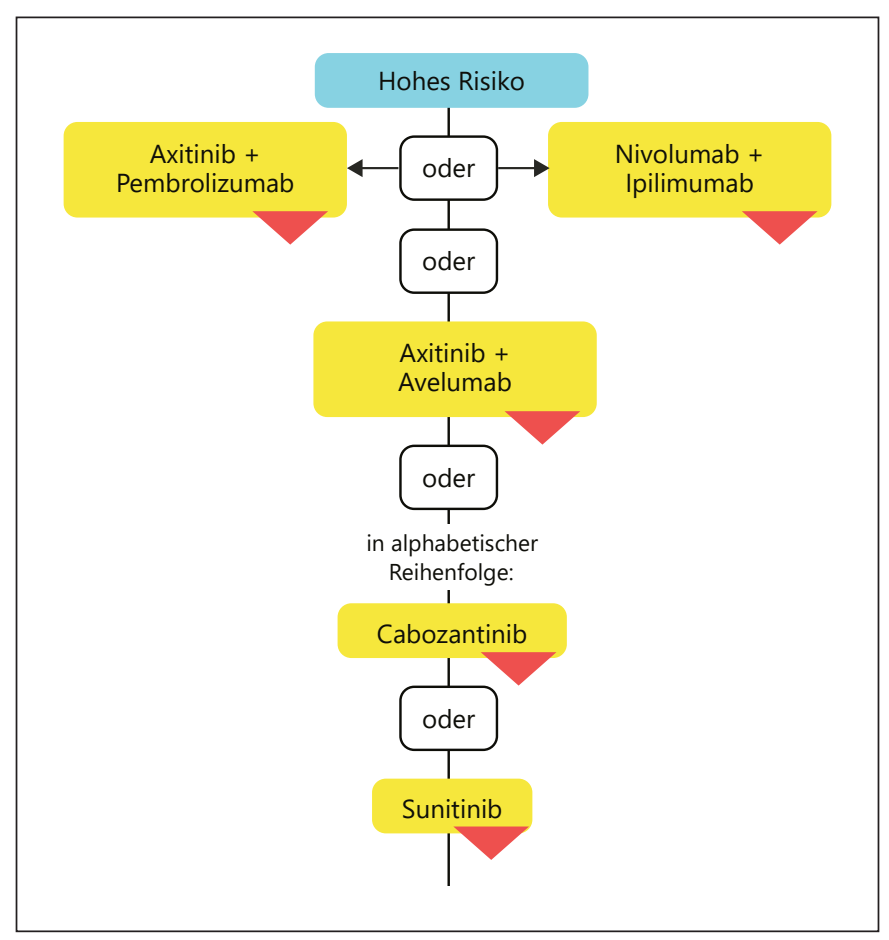

Abb. 3. Ausschnitt aus dem neu verlinkten Algorithmus für die medikamentöse Therapie des fortgeschrittenen/metastasierten Nierenzellkarzinoms unter www.onkopedia.com.

\section{Anpassungsbedarf bei seltenen Erkrankungen}

Eine wachsende Anzahl von Verfahren, die insbesondere die Hämatologie und Onkologie betreffen, entfällt auf Arzneimittel zur Behandlung seltener Erkrankungen (Orphan-Drug-Status). Für die Zuerkennung des Orphan-Drug-Status durch die Europäische ArzneimittelAgentur (EMA) dürfen weniger als 5 Personen pro 10.000 Einwohner an der betreffenden Erkrankung leiden, und befriedigende Behandlungsansätze dürfen nicht verfügbar sein. Der Orphan-Drug-Status eines Medikaments wird auch bei der frühen Nutzenbewertung eines Arzneimittels gesondert berücksichtigt: Für Arzneimittel, die zur Behandlung der seltenen Erkrankung zugelassen sind, gilt der medizinische Zusatznutzen als belegt. Auch entfällt die Festlegung einer zweckmäßigen Vergleichstherapie. Die Ausnahmeregelung gilt, solange eine Jahresumsatzgrenze von 50 Millionen Euro (einschließlich Umsatzsteuer) nicht überschritten wird [2].

Bei Betrachtung aller Verfahren zwischen 2012 und $2020 \mathrm{zu}$ Arzneimitteln mit Orphan-Drug-Status fällt auf,

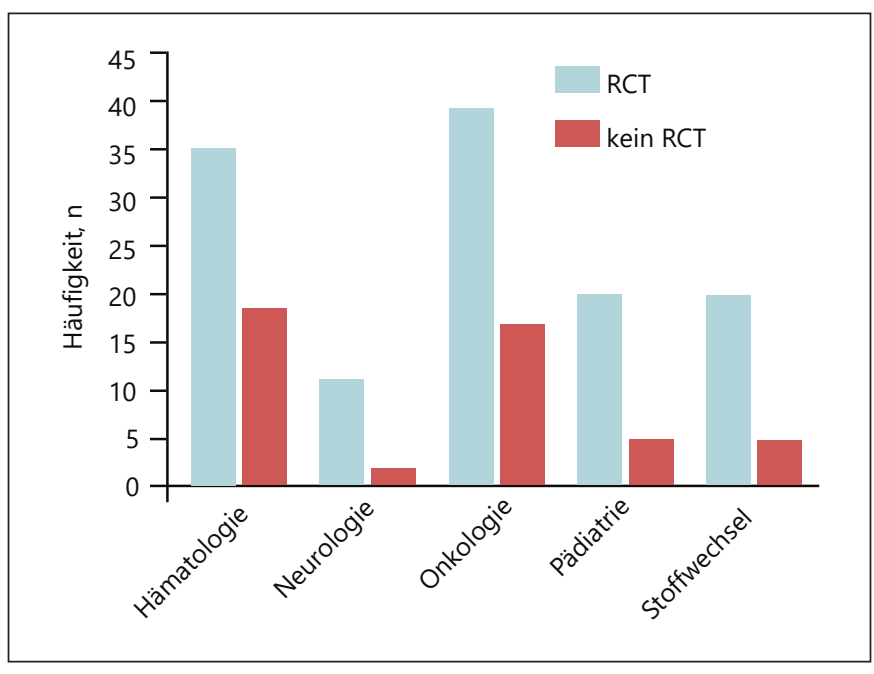

Abb. 4. Verfügbare Evidenzlage bei Arzneimitteln mit OrphanDrug-Status (2012-2020).

dass am häufigsten ein «nicht quantifizierbarer Zusatznutzen» zugesprochen wurde. Gleichzeitig wird deutlich, dass der Orphan-Drug-Status eines Arzneimittels nicht auch zwangsläufig bedeuten muss, dass keine Daten aus randomisierten kontrollierten Studien (randomized controlled trials, RCTs) vorhanden sind: Bei Wirkstoffen mit Orphan-Drug-Status überwiegt der Anteil der RCTs sowohl in der Hämatologie als auch in der Onkologie gegenüber Nicht-RCTs, sodass ein grundsätzlicher Verzicht auf eine reguläre frühe Nutzenbewertung nicht weiter gerechtfertigt erscheint (Abb. 4).

Weiterhin ist zu bedenken, dass sich der OrphanDrug-Status auf Basis der von der EMA angenommenen Prävalenz der Erkrankung nicht immer mit den Patient ${ }^{*}$ innenzahlen in Deutschland deckt. Ein entsprechendes Missverhältnis wird am Beispiel des multiplen Myeloms deutlich, das im Gegensatz zu den z.B. sehr kleinen, molekular definierten Subgruppen ohne OrphanDisease-Status innerhalb der Krankheitsentität des Lungenkarzinoms eher häufig ist [4]. Perspektivisch sind bei der frühen Nutzenbewertung von Orphan-Drug-Arzneimitteln die Kriterien zur Definition «seltener» bzw. «ultraseltener» Erkrankungen zu hinterfragen und besser abzubilden, gegebenenfalls die zu fordernden Signifikanzniveaus anzupassen und die Rolle der klinischen Studien wie die Machbarkeit von RCTs sowie Real-WorldDaten/Registerdaten zu überdenken. 


\section{AMNOG - ein Modell wird zum Standard Referent: Josef Hecken, Berlin}

Zehn Jahre nach der Beschlussfassung im Bundestag am 11.11.2010 lässt sich folgende Zwischenbilanz ziehen: Das «Gesetz zur Neuordnung des Arzneimittelmarktes in der gesetzlichen Krankenversicherung» (Arzneimittelmarkt Neuordnungsgesetz - AMNOG) hat sich fest etabliert und bewähren können: Die Zielsetzung, Arzneimittelpreise an ihren tatsächlichen Mehrwert zu koppeln, ist heute unter allen Verfahrensbeteiligten akzeptiert. Besonderes zugutegekommen ist dem AMNOG, dass es von Anfang an als «lernendes System» konzipiert wurde, das sich auf geänderte Rahmenbedingungen und aktuelle Herausforderungen einstellt. So konnte sich das
AMNOG im Zuge der stets offen geführten Diskussionen und im Verlauf auch nach einigen gesetzlichen Korrekturmaßnahmen zu einem Erfolgsmodell entwickeln (Tab. 1).

\section{Das AMNOG erfüllt seinen Zweck}

Bis zum 31.01.2021 wurden mehr als 270 verschiedene Arzneimittel in 454 Verfahren - abzüglich der aufgehobenen Beschlüsse - einer frühen Nutzenbewertung durch den Gemeinsamen Bundesausschuss (G-BA) unterzogen. Dabei blieben die Ergebnisse der Nutzenbewertung im Verlauf stabil: Mit ungefähr 57\% der abgeschlossenen Nutzenbewertungsverfahren wurde ein positiver Zusatznutzen häufiger beschieden, als es im internationalen Vergleich oftmals der Fall ist. Gleichzeitig konnten durch

Table 1. Weiterentwicklung des AMNOG: Meilensteine 2010-2020

\begin{tabular}{|c|c|c|}
\hline Jahr & Gesetz & Stichwort \\
\hline 2010 & $\begin{array}{l}\text { Arzneimittelneuordnungsgesetz (AMNOG) } \\
\text { Inkrafttreten: 1.1.2011 }\end{array}$ & Einführung der frühen Nutzenbewertung \\
\hline 2012 & $\begin{array}{l}\text { Zweites Gesetz zur Änderung arzneimittelrechtlicher } \\
\text { und anderer Vorschriften (2. AMGÄndG) } \\
\text { Inkrafttreten: } 25.10 .2012\end{array}$ & $\begin{array}{l}\text { - Neueinreichung von Dossiers möglich, sofern bei Ersteinreichung Unvollständigkeit } \\
\text { festgestellt wurde } \\
\text { - Beteiligung der Zulassungsbehörden bei Beratungen des G-BA zur Planung klinischer } \\
\text { Studien } \\
\text { - Berücksichtigung europäischer Preise in den Erstattungsbetragsverhandlungen }\end{array}$ \\
\hline 2013 & $\begin{array}{l}\text { Drittes Gesetz zur Änderung arzneimittelrechtlicher } \\
\text { und anderer Vorschriften (3. AMGÄndG) } \\
\text { Inkrafttreten: } 13.8 .2013\end{array}$ & $\begin{array}{l}\text { - Keine Klage gegen Bestandsmarktaufruf des G-BA } \\
\text { - Streichung des Wirtschaftlichkeitskriteriums bei Bestimmung der zweckmäßigen } \\
\text { Vergleichstherapie (zVT) }\end{array}$ \\
\hline 2014 & $\begin{array}{l}\text { Vierzehntes Gesetz zur Änderung des Fünften Buches } \\
\text { Sozialgesetzbuch (14. SGB V ÄndG) } \\
\text { Inkrafttreten: } 1.4 .2014\end{array}$ & - Bestandsmarktaufruf aufgehoben \\
\hline 2017 & $\begin{array}{l}\text { Gesetz zur Stärkung der Arzneimittelversorgung } \\
\text { in der GKV (GKV-Arzneimittelversorgungs- } \\
\text { stärkungsgesetz - AMVSG) } \\
\text { Inkrafttreten: } 13.5 .2017\end{array}$ & $\begin{array}{l}\text { - Antrag auf Freistellung nur vor Inverkehrbringen } \\
\text { - Ohne Zusatznutzen soll der Erstattungsbetrag die Kosten der ZVTnicht übersteigen } \\
\text { - Bündelung von Nutzenbewertungsverfahren } \\
\text { - Evidenztransfer bei PUMA-Arzneimitteln } \\
\text { - Resistenzsituation bei Antibiotika } \\
\text { - Bewertung von Bestandsmarktwirkstoffen, sofern ein neuer Unterlagenschutz zu } \\
\text { berücksichtigen ist } \\
\text { - Anpassung des EBM zeitgleich mit } ₫ 35 \text { a Beschluss } \\
\text { - Veröffentlichung einer maschinenlesbaren Fassung zur frühen Nutzenbewertung } \\
\text { - Erstattungsbetrag gilt auch für Krankenhäuser } \\
\text { - Festlegung von Kriterien für «Zusatznutzen gilt als nicht belegt» }\end{array}$ \\
\hline 2019 & $\begin{array}{l}\text { Terminservice- und Versorgungsgesetz (TSVG) } \\
\text { Inkrafttreten: 11.5.2019 }\end{array}$ & - Beschluss über die Nutzenbewertung auch in englischer Sprache \\
\hline 2019 & $\begin{array}{l}\text { Gesetz für mehr Sicherheit in der } \\
\text { Arzneimittelversorgung (GSAV) } \\
\text { Inkrafttreten: } 16.8 .2019\end{array}$ & $\begin{array}{l}\text { - Anwendungsbegleitende Datenerhebung } \\
\text { - ATMP-Qualitätssicherungsrichtlinie (\$136a SGB V) } \\
\text { - Stationäre Umsätze in Umsatzschwellenberechnung } \\
\text { - Beteiligung von Fachgesellschaften und AkdÄ } \\
\text { - Kostenausgleich mit Zulassungsbehörden }\end{array}$ \\
\hline 2020 & $\begin{array}{l}\text { Gesetz für einen fairen Kassenwettbewerb in der } \\
\text { gesetzlichen Krankenversicherung (GKV-FKG) } \\
\text { Inkrafttreten: } 1.4 .2020\end{array}$ & $\begin{array}{l}\text { - Erlass der anwendungsbegleitenden Datenerhebung frühestens zum erstmaligen } \\
\text { Inverkehrbringen } \\
\text { - ATMPs (außer Gewebeprodukte) werden im Rahmen des } \$ 35 \text { a bewertet } \\
\text { - Freistellung von Reserveantibiotika }\end{array}$ \\
\hline
\end{tabular}

Akdë, Arzneimittelkommission der deutschen Ärzteschaft; ATMPs, Advanced Therapy Medicinal Products; PUMA-Arzneimittel = Genehmigung für die pädiatrische Verwendung (Paediatric use marketing authorisation). 
das AMNOG 3,2 Milliarden Euro eingespart werden - bei wachsender Tendenz. Dabei hat das AMNOG seit 2012 einen durchschnittlichen Nutzenbewertungsrabatt von knapp über 20\% erzielt. Das im Gesetzesentwurf definierte Einsparziel von 2 Milliarden Euro wurde spätestens ab 2018 erreicht bzw. übertroffen, die dauerhaft realistische durchschnittliche jährliche Einsparung liegt inzwischen bei 3 Milliarden Euro.

Anders als initial befürchtet erwies sich die Sorge, dass die Markteinführung von patentgeschützten Arzneimitteln behindert würde, als unbegründet: Die mediane Latenz bis zur Verfügbarkeit eines neuen Arzneimittels betrug für den Zeitraum 2015-2018 in Deutschland lediglich 1,7 Monate - in Großbritannien dagegen 9,6 Monate und in Frankreich sogar 16,9 Monate. Damit werden Arzneimittel ab ihrer Zulassung durch die EMA derzeit auf dem deutschen Markt am schnellsten verfügbar. Auch in Bezug auf die verfügbare Gesamtzahl der Arzneimittel im Zulassungsjahr zeigte sich Deutschland noch vor Dänemark und Österreich führend [10]. Der zeitliche Vorsprung ist auf den Umstand zurückzuführen, dass der Betrag für die Erstattung eines neuen patentgeschützten Arzneimittels in den ersten 12 Monaten vom pharmazeutischen Unternehmen festgelegt wird, während der G-BA gleichzeitig innerhalb von 6 Monaten die Bewertung des Zusatznutzens gegenüber der zweckmäßigen Vergleichstherapie vornimmt. Diese Bewertung bildet die Grundlage für die anschließende Vereinbarung von Erstattungsbeträgen zwischen den gesetzlichen Krankenversicherungen (GKV-Spitzenverband) und dem pharmazeutischen Unternehmen. Weist der bewertete Wirkstoff keinen Zusatznutzen im Vergleich zur zweckmäßigen Vergleichstherapie auf, erfolgt entweder die Einordnung in eine Festbetragsgruppe oder der GKV-Spitzenverband vereinbart einen Erstattungsbetrag, bei dem die Jahrestherapiekosten nicht höher liegen sollen als die der zweckmäßigen Vergleichstherapie.

\section{Das AMNOG wird akzeptiert}

In einer webbasierten Stakeholder*innen-Befragung vergaben mehr als 70\% der Umfrageteilnehmer*innen die Note «gut» bzw. «sehr gut» und rund ein Viertel wertete den AMNOG-Prozess immerhin als «befriedigend.» Trotz eines gewissen Verzerrungspotenzials durch die vergleichsweise geringe Zahl der verwertbaren Antworten $(n=39)$ und den überproportional hohen Anteil an Krankenkassenvertreter ${ }^{*}$ innen bei der Umfrage ist das mehrheitlich positive Feedback zur frühen Nutzenbewertung beachtenswert: Zu den am häufigsten hervorgehobenen Charakteristika des AMNOG zählten Transparenz, Wissenschaftlichkeit, Fairness, Planbarkeit, Schnelligkeit und kooperatives Vorgehen [11].

Die (Informations-)Transparenz gilt auch im internationalen Vergleich als eines der herausragenden Merk- male des deutschen AMNOG-Verfahrens. So sind große Teile des Herstellerdossiers, alle Berichte des Instituts für Qualität und Wirtschaftlichkeit im Gesundheitswesen (IQWiG), die Stellungnahmen sowie das Wortprotokoll der mündlichen Anhörung und der G-BA-Beschluss öffentlich zugänglich [11].

\section{Orphan Drugs und GSAV}

Selbstverständlich bestehen nach wie vor Verbesserungspotenziale. Eines der aktuellen Handlungsfelder betrifft die Regelungen für neue Medikamente bei seltenen Erkrankungen («Orphan Drugs»), die nur einer eingeschränkten AMNOG-Nutzenbewertung unterliegen: Die gezielte Förderung von Orphan-Arzneimitteln ist gesetzlich verankert und ermöglicht eine Ausnahmeregelung, der zufolge der Zusatznutzen eines Orphan-Arzneimittels bereits durch die europaweite Zulassung als belegt gilt. Hinsichtlich Schnelligkeit und Verfügbarkeit ist Deutschland auch im Bereich der Orphan Drugs mit einer medianen Latenz von nur 1,3 Monaten zwischen EUZulassung und Markteinführung sowie $94 \%$ Verfügbarkeitsrate europaweit führend [10].

Allerdings hat der Anteil von Wirkstoffen, deren $\mathrm{Zu}$ satznutzen sich wegen der unzureichenden wissenschaftlichen Datenlage nicht quantifizieren lässt $(n=60 / 82$; Stand 21.1.2021), bei Arzneimitteln mit Orphan-DrugStatus über die Jahre zugenommen. Allein in 2019 verursachten Orphan Drugs 7,3\% des Bruttoumsatzes im GKV-Arzneimittelmarkt, obwohl sie nur 0,04\% des Verordnungsvolumens ausmachten [12].

Bei Überschreiten der jährlichen Umsatzschwelle von 50 Millionen Euro wird auch das Orphan-Arzneimittel der Nutzenbewertung unterzogen: Liegt zu diesem Zeitpunkt keine belastbare Evidenz (kein Anhaltspunkt für Zusatznutzen) vor, kann der Zusatznutzen im Zuge des regulären AMNOG-Verfahrens inklusive der Verhandlung zum Erstattungsbetrag aberkannt werden. Damit ist auch das Absinken der Therapiekosten auf das Niveau des Behandlungsstandards (in der Regel «Best Supportive Care») verbunden [11].

Einen neuen gesetzgeberischen Ansatz, Evidenzlücken von Orphan Drugs zu adressieren, liefert das am 16.08.2019 in Kraft getretene «Gesetz für mehr Sicherheit in der Arzneimittelversorgung»(GSAV): Damit erhält der G-BA die Option, vom pharmazeutischen Unternehmen innerhalb einer angemessenen Frist weitere anwendungsbegleitende Datenerhebungen und Auswertungen zur Zusatznutzenbestimmung zu verlangen [13]. Dies bezieht sich auf - Arzneimittel mit bedingter Zulassung, bei denen die Zulassung bei noch unvollständiger klinischer Datenlage erteilt wurde (z.B. Arzneimittel, die zur Behandlung, Vorbeugung oder ärztlichen Diagnosestellung von Erkrankungen bestimmt sind, die zu schwerer Invalidität führen oder lebensbedrohlich sind), 


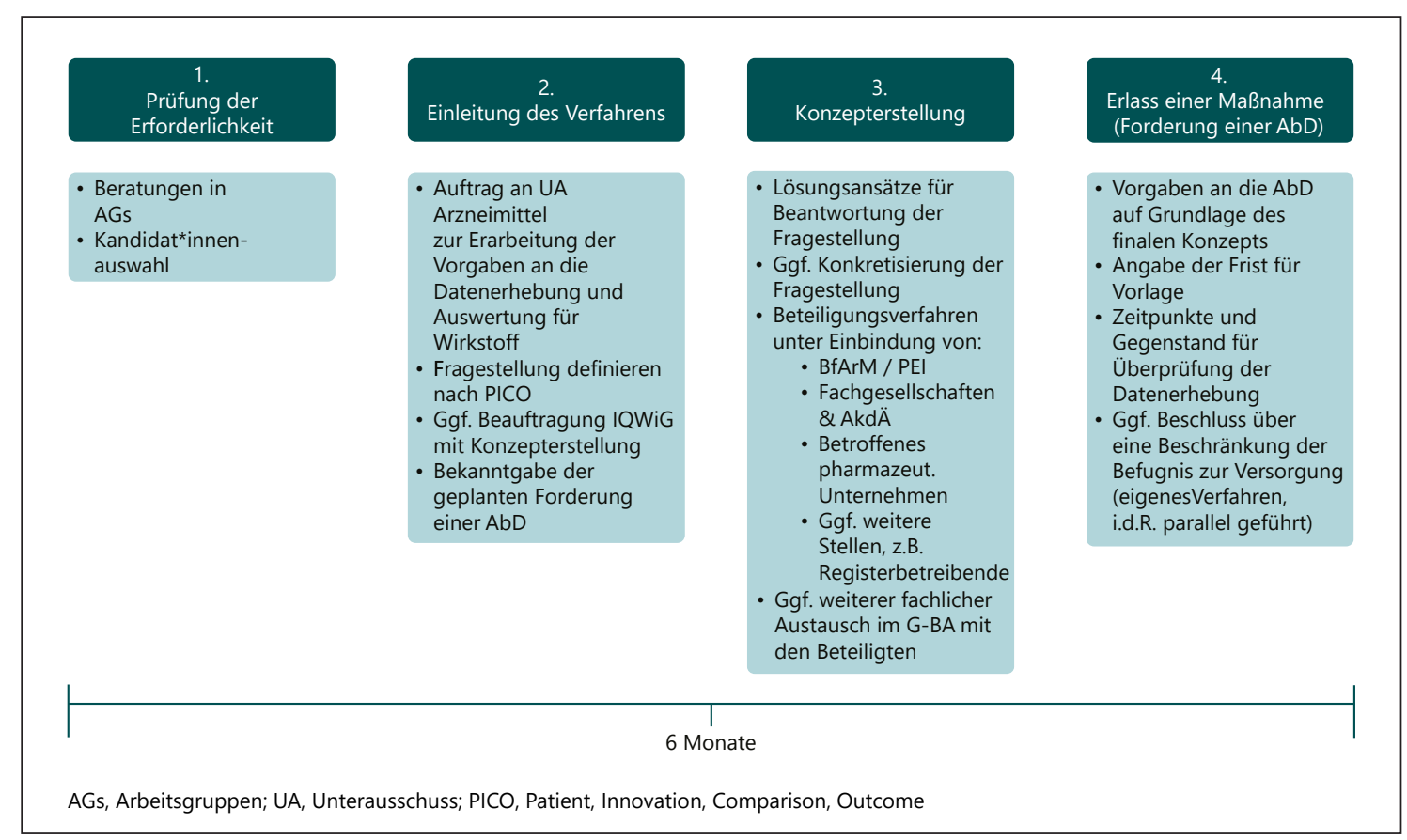

Abb. 5. Verfahrensschritte der anwendungsbegleitenden Datenerhebungen (AbD) im Überblick.

- Arzneimittel, deren Zulassung unter außergewöhnlichen Umständen erfolgt ist (z.B. ist das Anwendungsgebiet so selten, dass sich keine umfangreiche Evidenz zur Wirksamkeit und Sicherheit anfordern lässt, oder die Durchführung von randomisierten kontrollierten Studien ist aus ethischen Gründen nicht möglich) sowie

- Arzneimittel für seltene Erkrankungen (Orphan Drugs).

Wenn auch nicht explizit aufgeführt, können auch Arzneimittel für neuartige Therapien (Advanced Therapy Medicinal Products, ATMPs) betroffen sein, bei denen es sich in der Mehrzahl um Orphan Drugs handelt (z.B. CAR-T-Zell-Therapien - CAR = chimärer Antigenrezeptor - und Gentherapeutika). Bei der Gentherapie mit Onasemnogen-Abeparvovec (Zolgensma), für die in 2020 eine bedingte Marktzulassung zur Behandlung der spinalen Muskelatrophie ausgesprochen wurde, hat der G-BA erstmals die Erforderlichkeit einer anwendungsbegleitenden Datenerhebung festgestellt: Der Schritt ist damit begründet, dass keine aussagekräftigen Daten für die Beurteilung des langfristigen Nutzens oder Schadens der Behandlung vorliegen und vergleichende Daten zu Therapiealternativen fehlen [14].

Das Verfahren zur Forderung einer anwendungsbegleitenden Datenerhebung kann frühestens zum Zeitpunkt des erstmaligen Inverkehrbringens des entsprechenden Arzneimittels verlangt werden und ist in 4 Verfahrensschritte gegliedert (Abb. 5):
- Prüfung der Erforderlichkeit einer Datenerhebung: Für die Beurteilung, ob eine Datenerhebung erforderlich ist, werden neben Informationen aus Studienberichten unter anderem Informationen über die möglichen Evidenzlücken aus dem Zulassungsverfahren des Arzneimittels herangezogen, die der G-BA im fachlichen Austausch mit dem Bundesinstitut für Arzneimittel und Medizinprodukte (BfArM) und dem Paul-Ehrlich-Institut (PEI) erhält. Wird die Erforderlichkeit einer Datenerhebung festgestellt, erfolgt die Beauftragung des Unterausschusses Arzneimittel mit der Vorbereitung eines Konzeptentwurfs. Dieser sollte grundsätzlich innerhalb von 6 Monaten abgeschlossen sein.

- Einleitung des Verfahrens: Mit der Bekanntgabe der geplanten Forderung einer anwendungsbegleitenden Datenerhebung erstellen der G-BA oder das IQWiG die wissenschaftliche Ausarbeitung des Konzeptentwurfs. Darin sollen die Anforderungen an Art, Dauer und Umfang der Datenerhebung, die Fragestellung, die Gegenstand der Datenerhebung und Auswertungen sein soll, einschließlich der zu erfassenden patientenrelevanten Endpunkte und die Methodik der Datenerhebung sowie der Auswertung durch das pharmazeutische Unternehmen enthalten sein.

- Vorbereitung des Konzepts für die Anforderungen an eine anwendungsbegleitende Datenerhebung und Beteiligung sachverständiger Stellen: Der Konzeptentwurf wird den zu beteiligenden Stellen (unter anderem den Bundesoberbehörden BfArM und PEI, den wissen- 


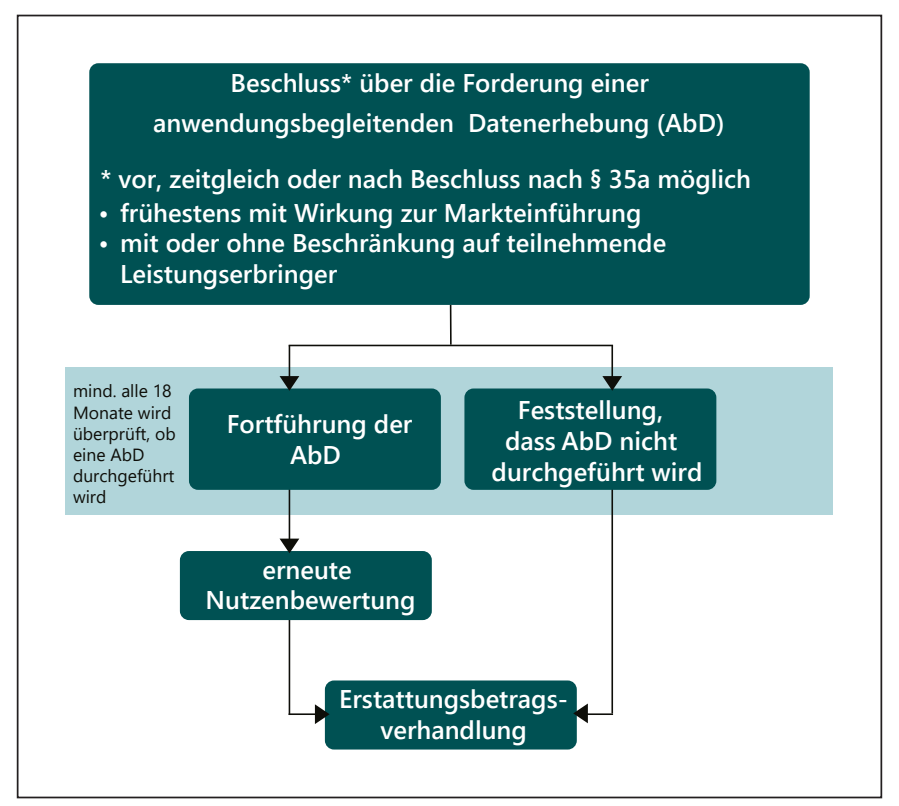

Abb. 6. Ablauf der anwendungsbegleitenden Datenerhebungen.

schaftlich-medizinischen Fachgesellschaften, der AkdÄ, sowie den betroffenen pharmazeutischen Unternehmen) zugeleitet. Ihre schriftliche Äußerung zum Konzeptentwurf sollen die zu beteiligenden Stellen innerhalb von 4 Wochen an den G-BA übersenden.

- Beschluss über die Forderung einer anwendungsbegleitenden Datenerhebung: Per Plenumsbeschluss legt der G-BA fest, ob er vom pharmazeutischen Unternehmen eine anwendungsbegleitende Datenerhebung und Auswertungen fordert. Er legt darin die Vorgaben an die anwendungsbegleitende Datenerhebung und die Auswertungen anhand des zuvor erstellten Konzepts fest. Darüber hinaus bestimmt er die Zeitpunkte für die Überprüfung der Datenerhebung und für die Abgabe der Auswertungen in Form eines Dossiers, die für die Durchführung einer erneuten Nutzenbewertung benötigt werden. Durch eine Einschränkung der Versorgung mit diesen Arzneimitteln auf Leistungserbringer, die an der geforderten anwendungsbegleitenden Datenerhebung mitwirken, soll eine möglichst vollständige und valide Datenerhebung unterstützt werden. Der G-BA überprüft in regelmäßigen Abständen, mindestens jedoch alle 18 Monate, ob die Datenerhebung durchgeführt wird/nicht mehr durchgeführt werden kann, ob sie hinreichende Belege für eine erneute Nutzenbewertung erbringen wird, oder ob Bedarf für Anpassungen an die Vorgaben des Beschlusses besteht (Abb. 6).

Wichtig ist ein möglichst frühzeitiger Beginn der Datenerhebung, die nach Möglichkeit auch vollständig bzw. vollzählig durchzuführen ist. Die anwendungsbegleitende Datenerhebung schließt auch Registerstudien ein, die eine repräsentative Stichprobe der Patient*innenpopulation umfassen sollten. Optimalerweise sollte die Datenerhebung bereits vor dem geplanten Marktzugang einsetzen (Kontrollgruppe), patient*innenrelevante Endpunkte sollten miterfasst sein (insbesondere Krankheitssymptomatik und Lebensqualität), und die Registerorganisation sollte durch unabhängige Institutionen vorgenommen werden (Kasten 1). Um auch über längere Zeiträume eine sorgfältige Datenerhebung bei angemessener Datenqualität zu gewährleisten, bedarf es zudem sicherlich einer gesicherten Finanzierung der Register und zusätzlicher Anreize für die Ärzteschaft und Betroffene, sich an Registerstudien zu beteiligen.

\footnotetext{
Wesentliche Anforderungen an Register

- Generierung von vergleichender Evidenz muss gewährleistet sein - Mit indikationsspezifischen Registern werden auch Daten alternativ behandelter Patient*innen erfasst

- Beginn der Datenerhebung optimalerweise vor dem Marktzugang (Kontrollgruppe)

- Es sollten Interventionen einschließlich Komparatoren beschrieben sein

- Erhebung von patient*innenrelevanten Endpunkten (insbesondere zur Krankheitssymptomatik und Lebensqualität)

- Kompatibilität mit bestehenden Registern (Doppelerfassungen vermeiden) und Erweiterbarkeit (zur Kombination mit ergänzender studienindividueller Datenerhebung)

\section{Herausforderungen:}

- Organisation der Register durch unabhängige Institutionen - Verbindliche Finanzierung der Register mit Beteiligung der Industrie - Vollständigkeit, Geeignetheit für Nutzenbewertung, Erfahrungen - Anreize schaffen für Ärzt*innen, Patient*innen sowie Organisationen für die Register-Teilnahme
}

Kasten 1 Anwendungsbegleitende Datenerhebungen: Hinweise zur Ausgestaltung von Registern 
Abb. 7. Zunehmende AMNOG-generierte Einsparungen im Verlauf; mod. nach [15].

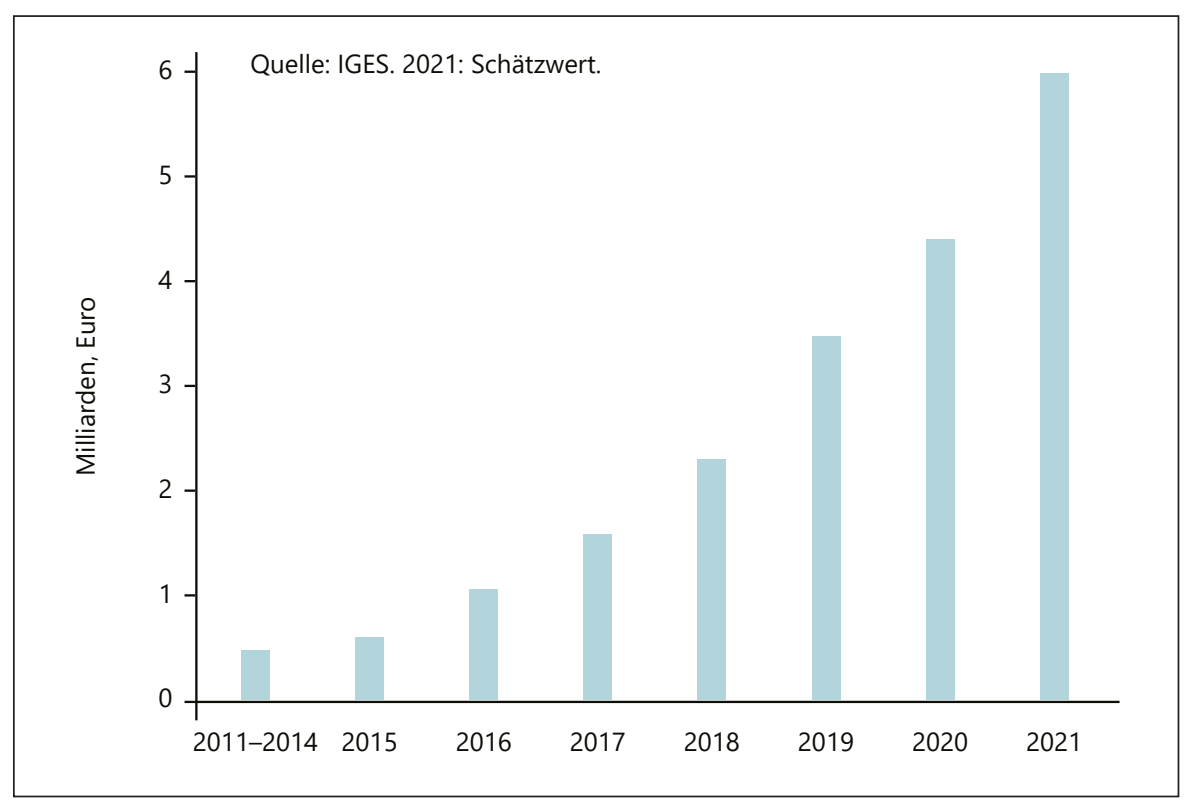

\section{Innovationsstandort Deutschland: AMNOG and beyond? Referent: Han Steutel, Berlin}

Mit der Einführung der Zusatznutzenbewertung gemäß dem AMNOG im Jahr 2011 für das Segment der innovativen Arzneimittel und den darauf basierenden Erstattungsbeträgen wurde die letzte Lücke der Erstattungsregelungen in der Arzneimittelversorgung der Gesetzlichen Krankenversicherung (GKV) geschlossen. Das AMNOG hat über die Jahre Einsparungen generiert, die sich auf eine Entlastung der Kassen um insgesamt 14 Milliarden Euro kumuliert haben. Allein für 2021 werden Einsparungen von etwa 6 Milliarden Euro erwartet (Abb. 7) [15]. Doch nicht nur die Sparbilanz zum AMNOG kann sich sehen lassen: Auch der Anteil der abgeschlossenen Zusatznutzenbewertungsverfahren stieg im Verlauf an und hat mit 32 neuen Wirkstoffen im Jahr 2020 ein neues Rekordhoch erreicht. Das AMNOG-Verfahren hat zudem dazu geführt, dass die in den späten 2000-er Jahren geäußerte Kritik an Analogpräparaten («Me-too»-Produkte) verstummt ist.

Die hohe Innovationsbereitschaft zeichnet sich derzeit besonders deutlich bei der Entwicklung von Arzneimitteln im Indikationsbereich der Onkologie ab: Rund 30\% der innovativen Wirkstoffe, die 2020 das Zusatznutzenbewertungsverfahren durchliefen, adressierten eine Krebserkrankung [11]. Und: In rund 80\% der Fälle konnte für Onkologika auch der Nachweis eines Zusatznutzens erbracht werden (vfa-AMNOG-Verfahrensdatenbank, Stand: 28.01.2021; vfa = Verband Forschender Arzneimittelhersteller e.V.).
Wege aus der NUB-Lücke

Mit den rasanten Fortschritten in der Entwicklung von neuartigen Arzneimitteltherapien, die vorwiegend im Krankenhaus eingesetzt werden, ergeben sich spezifische Herausforderungen in der Vergütung dieser Produkte, die einer Optimierung bzw. Anpassung bedürfen, damit sie bei Patient*innen zum Einsatz kommen können. So sollten innovative Arzneimittel nach ihrer Marktverfügbarkeit auch zügig in der Patient ${ }^{*}$ innenversorgung ankommen - sowohl ambulant als auch stationär. Für im stationären Versorgungsbereich eingesetzte Arzneimittel gelten allerdings völlig andere Erstattungsregeln als im ambulanten Bereich. Nach dem DRG-Verfahren werden innovative Arzneimittel im stationären Einsatz überwiegend in die Kategorie der «neuen Untersuchungs- und Behandlungsmethoden» (NUB) eingeordnet. Dabei kann sich derzeit eine unklare Vergütungssituation ergeben, die sogenannte «NUB-Lücke».

Seit 2018 werden innovative Arzneimittel wie z.B. CAR-T-Zell-Therapien zwar von der AMNOG-Zusatznutzenbewertung erfasst. Jedoch werden diese Innovationen nicht stets zugleich auch entsprechend vergütet [11]. Da das dafür am Institut für das Entgeltsystem im Krankenhaus (InEK) angesiedelte Verfahren vergleichsweise umständlich und zeitaufwendig ist, kann es bis zu 3 oder gar 5 Jahre dauern, bis die Refinanzierung für die neue Behandlung gesichert ist. Bevor die neue medizinische Leistung Patient ${ }^{*}$ innen zuteil wird, kann es folglich zu einer jahrelangen Verzögerung kommen, was Deutschland hinter andere EU-Länder zurückwirft.

Eine engere Orientierung des NUB-Verfahrens am AMNOG könnte künftig zu einer rascheren Vergütungs- 


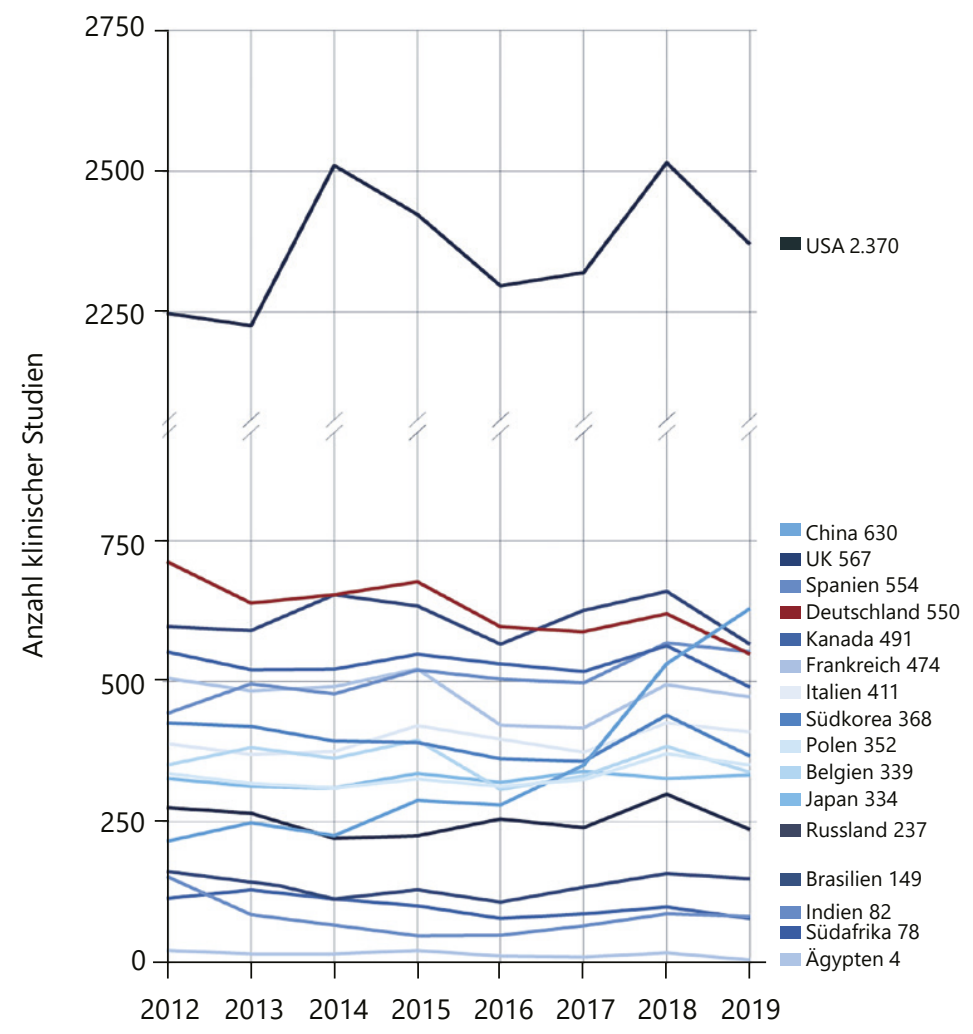

tionalen Vergleich; mod. nach [16].

und Versorgungssicherheit für innovative Therapieansätze in den Kliniken beitragen. Insbesondere für hochkomplexe Einmaltherapien wie eine Gentherapie, die sich an kleine Patient*innengruppen richtet, könnten zudem ergänzend flexiblere Erstattungsmodelle sinnvoll sein: Der «Pay-for-Performance»-Ansatz koppelt die Vergütung eines Arzneimittels beim einzelnen Patienten an den individuellen Therapieerfolg. Der Ansatz könnte dazu beitragen, eine bessere Verteilung des Finanzierungsrisikos zu erreichen und potenzielle Ergebnisunsicherheiten bei der Langzeitwirkung von neuen Therapien zu verringern. Dazu braucht es mehr Verhandlungsspielraum der einzelnen Krankenkassen und weniger Machtfülle beim GKV-Spitzenverband.

Gegenwärtig arbeitet die Europäische Union (EU) an einer gesetzlichen Grundlage zur Durchführung einer gemeinsamen klinischen Nutzenbewertung von Arzneimitteln, auch EU-HTA (Health Technology Assessment) genannt. Hierdurch bietet sich die Chance, Doppelstrukturen innerhalb der EU abzubauen und etablierte Qualitätsstandards zu sichern. Eine verbesserte Zusammenarbeit zur Evidenzgenerierung zwischen der europäischen Zulassungsbehörde und den verantwortlichen HTA-Institutionen unter Einbindung der pharmazeuti- schen Unternehmens ist dabei unabdingbar. So kann ein europäischer Nutzenbewertungsprozess - transparente Verfahrensabläufe und die vollständige Einbeziehung der verfügbaren Studiendaten vorausgesetzt - künftig den Zugang zu Therapieoptionen für Patient*innen auf europäischer wie nationaler Ebene weiter ausbauen und verbessern, ohne dabei die positiven Aspekte des deutschen Gesundheitssystems infrage zu stellen.

\section{Innovationsstandort Deutschland}

Als überaus erfreulich ist der Stellenwert von Deutschland als Innovationsstandort für die Pharmaforschung, -entwicklung und -produktion zu konstatieren. Wie die innerhalb von nur 10 Monaten in Rekordzeit gelungene mRNA-basierte COVID-19-Impfstoffentwicklung beispielhaft zeigt, hat Deutschland herausragendes Potenzial in der medizinischen Biotechnologie: So hat das Mainzer Biotech-Unternehmen BioNTech zusammen mit dem Unternehmen Pfizer den weltweit ersten mRNA-Impfstoff gegen das SARS-CoV-2-Virus auf den Markt gebracht. Das Tübinger Unternehmen CureVac erwartet in diesem Bereich die europäische Zulassung bis Ende Juni 2021 (Stand der Information: Mai 2021). Das Beispiel macht deutlich, dass hierzulande nicht nur in der Grund- 
lagenforschung und Hochtechnologie eine herausragende Infrastruktur von universitären und nicht universitären Forschungseinrichtungen sowie innovativen Pharmaund Biotech-Firmen geboten wird, sondern auch bei den Produktionsstätten eine hervorragende Basis für BiotechInnovationen «Made in Germany» besteht.

Eine überaus wichtige Rolle bei der Translation von medizinischer Forschung in die klinische Praxis kommt klinischen Studien zu - idealerweise über die ganze Bandbreite der klinischen Studienphasen hinweg. Trotz des hohen Stellenwerts, den Deutschland als Studienstandort für die internationalen forschenden Pharma-Unternehmen einnimmt, lässt sich bei der Zahl der klinischen Stu- dien ein Abwärtstrend beobachten: Deutschland war lange bei der Zahl der klinischen Prüfungen die Nummer 1 in Europa (weltweit Nummer 2 hinter den USA). In 2017 wurde Deutschland von Großbritannien überholt und 2019 zudem noch von Spanien; Deutschland liegt inzwischen weltweit nur noch auf dem fünften Platz (Abb. 8) [16]. Als wichtigster Wettbewerbsnachteil erweisen sich oftmals die bürokratischen Hürden, die zu teilweise sehr langen Genehmigungsverfahren für klinische Studien und auch zu föderal unterschiedlichen Anforderungen führen. Hier sind attraktivere Rahmenbedingungen gefragt, damit Deutschland den internationalen Anschluss an die Spitzenstandorte nicht verliert.

\section{Ambulante Versorgung \\ Ambulante Versorgung von Tumorpatient*innen in Deutschland \\ Referent: Ingo Tamm, Berlin}

Der Versorgungsbedarf von Tumorpatient ${ }^{*}$ innen ist nach wie vor hoch: Bis zum Jahr 2025 wird mit einer Zunahme der jährlich neu erkrankten Krebspatient*innen auf schätzungsweise 523.000 Fälle in Deutschland gerechnet [17]. Als Hauptgrund für die Entstehung von Krebs gilt die demografische Entwicklung mit der gestiegenen Lebenserwartung und Lebensstilfaktoren. Daneben geht die Zunahme der Neuerkrankungsraten zum Teil auch auf die verbesserte Früherkennungsdiagnostikzurück. Gleichzeitigermöglichen Fortschritte der Diagnostik und Behandlung sowie die verbesserte interdisziplinäre Zusammenarbeit in den Zentren der onkologischen Versorgung eine sinkende Krebsmortalität. Im europäischen Vergleich liegt Deutschland bei der Inzidenz etwas oberhalb und bei der krebsbedingten Mortalität unter dem altersstandardisierten Mittelwert [18].

\section{Flächendeckende Versorgung durch}

\section{Schwerpunktpraxen}

Die onkologischen Schwerpunktpraxen übernehmen in Deutschland einen bedeutenden Anteil an der Versorgung von Tumorpatient*innen: Sie bilden ein feinmaschiges Netzwerk, das jährlich etwa 650.000 Patient*innen mit einer soliden oder hämatologischen Tumorerkrankung wohnortnah und flächendeckend ambulant versorgt [18]. Eine onkologische Schwerpunktpraxis versorgt im Median 1.563 Patient*innen (bezogen auf das 3. Quartal 2018). Die Anzahl der versorgten Patient*innen je Praxis ist im Vergleich zu 2008 um etwa 54\% gestiegen, wobei die Praxen heute selten als Einzelpraxis geführt sind und an den Schwerpunktpraxen durchschnittlich 3,8 Fachärzt*innen beschäftigt sind $[18,19]$.

Die niedergelassenen Hämatolog*innen und Onkolog ${ }^{\star}$ innen sind bundesweit als Netzwerk im Berufsverband der Niedergelassenen Hämatologen und Onkologen in Deutschland e.V.(BNHO) organisiert. Zusätzlich sind die meisten Fachärzt ${ }^{*}$ innen dieser Berufsgruppe knapp 200 - partnerschaftlich mit dem Wissenschaftlichen Institut der Niedergelassenen Hämatologen und Onkologen (WINHO) verbunden. Während der BNHO die Interessen von derzeit etwa 600 Ärzt $^{*}$ innen in über 360 onkologischen Schwerpunktpraxen vertritt, ermöglicht das WINHO industrieunabhängige Forschungsprojekte, die eine verbesserte Patient ${ }^{*}$ nnenversorgung zum Ziel haben (Kasten 2). Darüber hinaus setzen sich sowohl der BNHO als auch das WINHO für die Gewährleistung von Qualität und Transparenz in den hämatologischen und onkologischen Schwerpunktpraxen ein. Der regelmäßig veröffentlichte Qualitätsbericht ist öffentlich zugänglich (www.winho.de).

WINHO - Wissenschaftliches Institut der Niedergelassenen Hämatologen und Onkologen

Das WINHO wurde 2004 vom BNHO gegründet und wird durch Mitgliedsbeiträge der niedergelassenen Ärzt*innen sowie durch öffentliche, projektspezifische Drittmittel finanziert.

Die Drittmittel werden unter anderem beim Bundesministerium für Gesundheit, beim Bundesministerium für Bildung und Forschung, bei der Deutschen Krebshilfe, der Deutschen Forschungsgemeinschaft, der Deutschen Rentenversicherung sowie beim Zentralinstitut für die kassenärztliche Versorgung eingeworben.

Auf diese Weise ermöglichen niedergelassene Hämatolog*innen und Onkolog*innen eine von der pharmazeutischen Industrie unabhängige Forschung - mit Schwerpunkt auf die verbesserte Versorgung der Patient*innen.

Kasten 2 Hintergrundinformation zum WINHO; mod. nach [18]. 


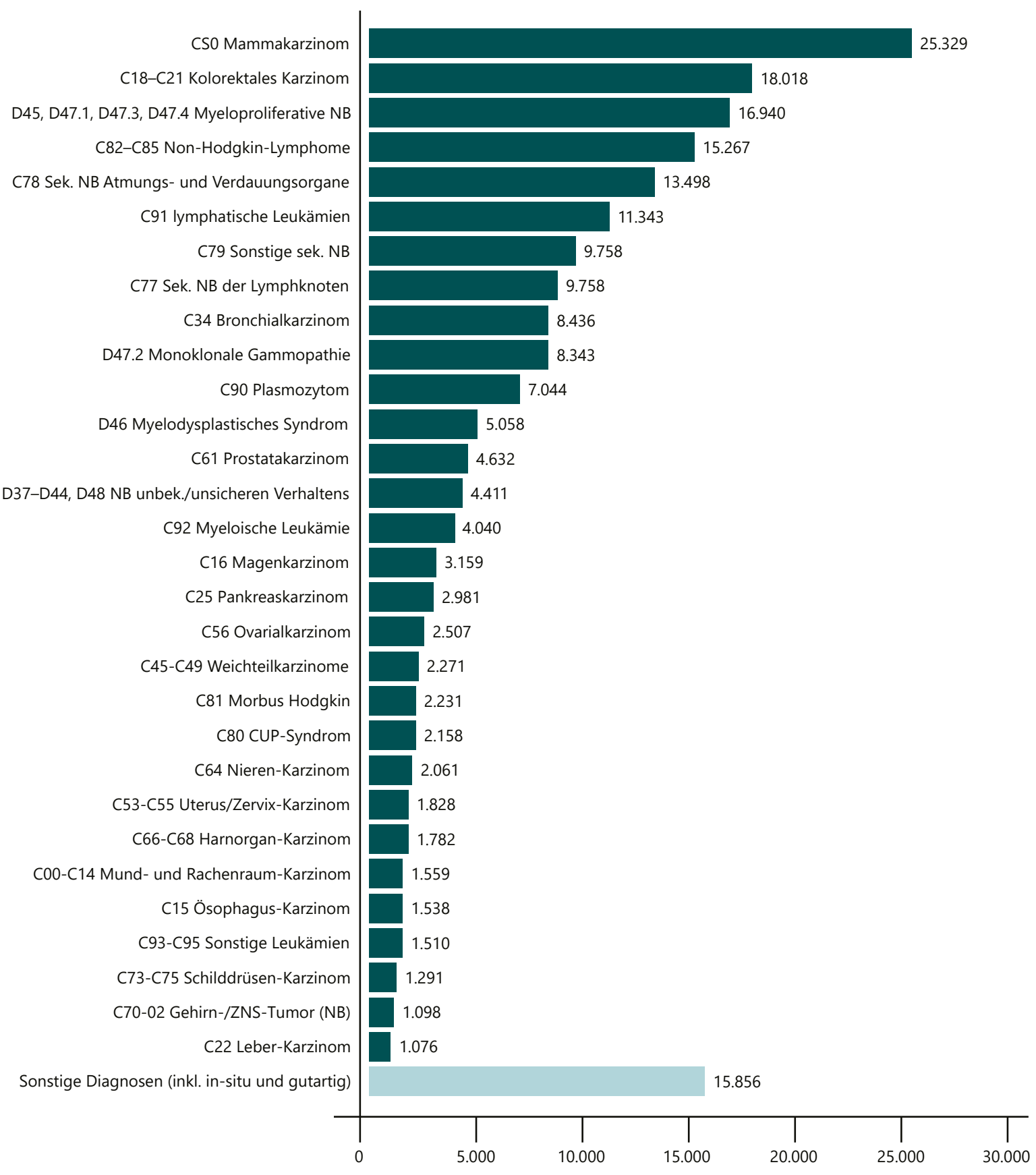

205.943 Diagnosen bei 199.463 Patient*innen aus 109 Praxen

Abb. 9. Niedergelassene Hämatolog^innen und Onkolog*innen: Häufige Diagnosen im 3. Quartal 2018 nach International Classification of Diseases 10 (ICD-10); mod. nach [18]. 


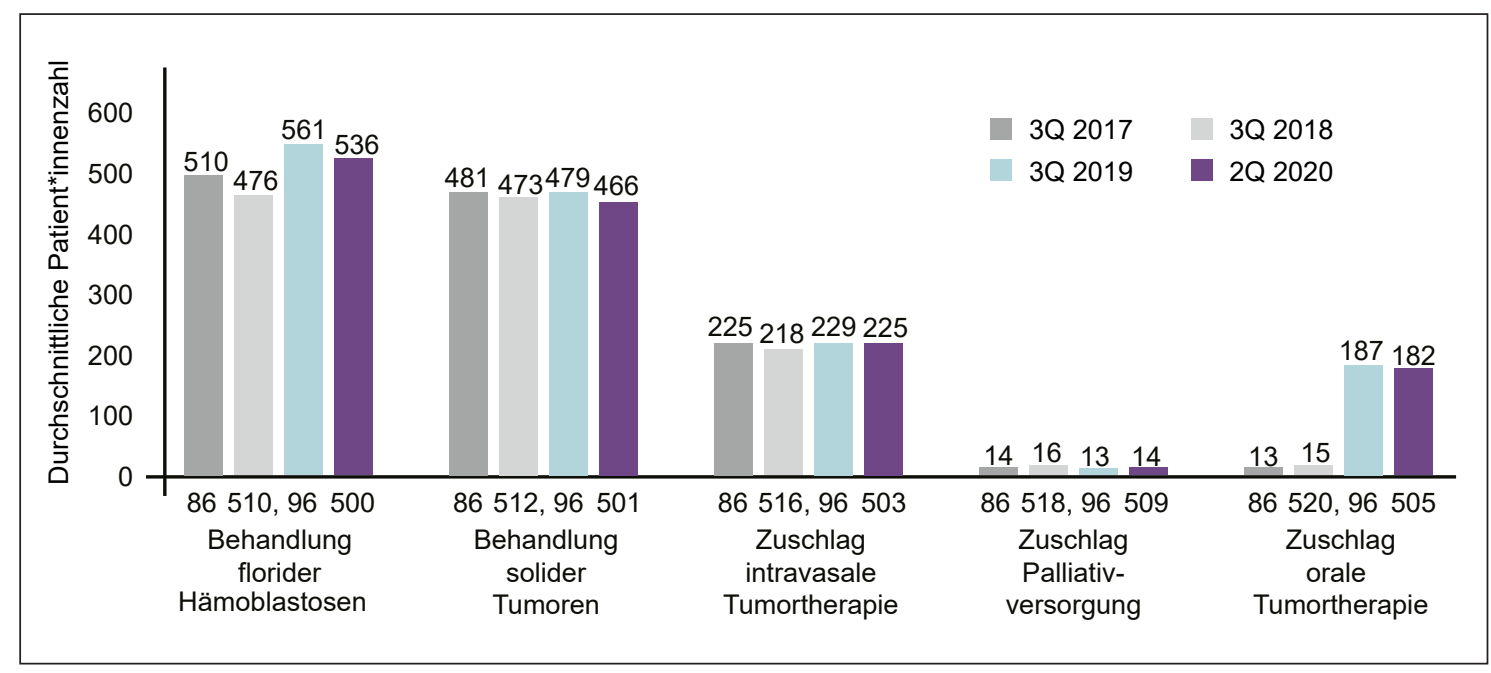

Abb. 10. Stabilität in der ambulanten Versorgung onkologischer Patient*innen: Aufschlüsselung der durchschnittlichen Patient*innenzahl pro OV-Ziffer und Praxis 2017-2020 (OV, Onkologie-Vereinbarung); mod. nach [20].

\section{Stabile Versorgung unter Pandemiebedingungen}

Die ambulante Versorgung in den onkologischen Schwerpunktpraxen deckt ein breites Spektrum an Krebserkrankungen $\mathrm{ab}$ - darunter am häufigsten Mammakarzinome, kolorektale Karzinome und myeloproliferative Tumorerkrankungen (Abb. 9) [18]. Wie tragfähig die derzeitige Versorgungsstruktur für onkologische Patient ${ }^{\star}$ innen selbst unter COVID-19-Pandemiebedingungen ist, zeigt eine aktuelle Auswertung aus 101 Schwerpunktpraxen, die im zweiten Quartal 2020 bundesweit etwa 162.000 Patient*innen versorgt haben: Bei der detaillierten Aufschlüsselung der OV-Ziffern (OV = Onkologie-Vereinbarung) zeichnet sich ab, dass die seit Jahren auf ähnlichem Niveau liegende, stabile Kontinuität in der ambulanten Versorgung von Tumorpatient*innen auch unter pandemischen Bedingungen sichergestellt wird (Abb. 10). So kam es zu keinem Zeitpunkt zu einer Unterversorgung der Patient*innen in den onkologischen Schwerpunktpraxen [20]. Der Anteil der therapeutisch betreuten Patient*innen mit einer floriden Tumorerkrankung erhöhte sich im Vergleich zu den 3 vorangegangenen Jahren im Mittel um 8,3\%. Als mögliche Erklärung wird aufgeführt, dass sich Versorger im stationären Bereich während der ersten Pandemiewelle im Frühjahr 2020 auf das Vorhalten von personellen und strukturellen Ressourcen einstellen mussten. In diesem Fall trug die intersektorale Kooperation zur Sicherstellung der verschiedenen Versorgungsaufträge von sowohl von Intensivpatient*innen (stationär) als auch Krebspatient*innen (ambulant) bei. Gleichzeitig wurde die elektive Diagnostik angepasst, zeitlich unkritische (verlaufs)diagnostische Untersuchungen bei stabil-chronischen Patient*innen wurden verschoben und ein Teil der Sprechstunden wurde als Videokonferenz abgehalten.
Insgesamt konnte die sektorale Versorgungsstruktur ihre hohe Anpassungsfähigkeit unter Beweis stellen und zu einer Entlastung des stationären Sektors beitragen - und dies trotz der pandemiebedingt erschwerenden Bedingungen: Denn im Vergleich zum Vorjahr nahm der bürokratische Aufwand durch die gesteigerten Nachweisund Berichtspflichten nochmals zu. Durch die erweiterten Hygieneauflagen wurden Anpassungen der Abläufe im Praxisalltag erforderlich und für die organisatorische Bewältigung mussten EDV-technische Aufrüstungen sowie aufgrund des dynamischen Pandemiegeschehens noch fortlaufend weitere Anpassungen beim Hygieneund Testkonzept von Patient ${ }^{\star}$ innen und Personal vorgenommen werden [20].

\section{Preisentwicklung für vertragsärztliche Leistungen}

Bei der Frage, wie sich die Preise für Leistungen der Vertragsärzt ${ }^{*}$ innen im Vergleich zum Krankenhausbereich oder zur generellen Lohnsteigerung entwickelt haben, fiel die Antwort einer Untersuchung des Zentralinstituts für kassenärztliche Versorgung $(\mathrm{Zi})$ ernüchternd aus: Während der Nominallohnindex aller Angestellten in Deutschland zwischen 2010 und 2017 um 19\% anstieg und der Tarifvertrag für leitende Oberärzt ${ }^{*}$ innen im Krankenhaus - Sonderentgelte nicht eingerechnet - eine Gehaltssteigerung von 15\% herbeiführte, betrug der $\mathrm{Zu}-$ wach beim Orientierungswert für die Vertragärzt ${ }^{\star}$ innen und Psychotherapeut ${ }^{*}$ innen lediglich 6\% (Abb. 11). Der Orientierungswert ist für die Entwicklung der Vergütung im vertragsärztlichen Bereich maßgeblich und entspricht dem Punktwert, der jährlich auf Bundesebene durch den Bewertungsausschuss und die Vereinbarungen der Gesamtvertragspartner festgelegt wird [21, 22].
Wörmann et al. 


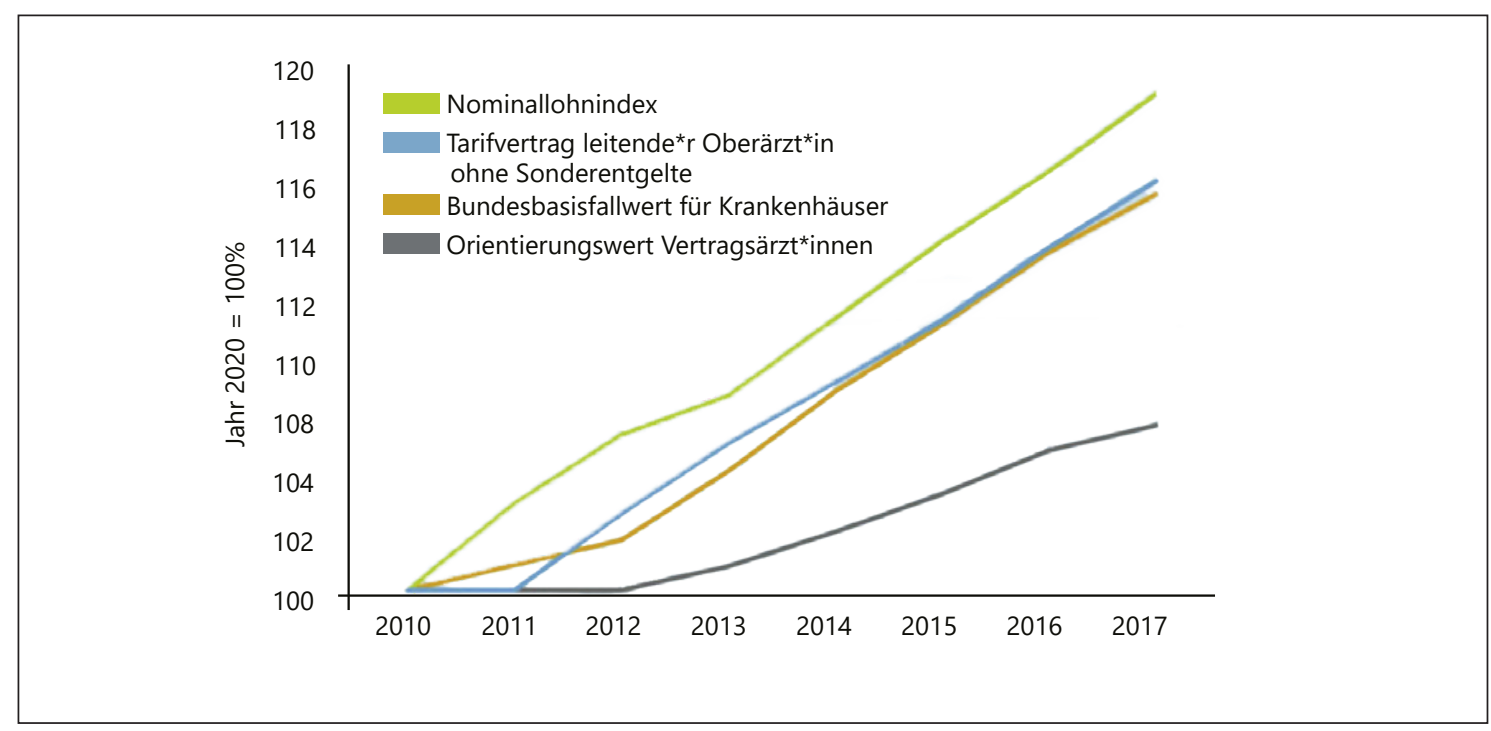

Abb. 11. Entwicklung des Orientierungswerts für ärztliche Leistungen (2010-2017) im Vergleich; mod. nach $[21]$.

Bei der Betrachtung der Betriebskosten in der vertragsärztlichen Versorgung fällt seit einigen Jahren ein starker Anstieg der Praxisaufwendungen für das Personal auf: Allein im Zeitraum 2015-2018 machten die Aufwendungen für Personal den stärksten absoluten Anstieg aller Ausgabekategorien aus - mit einer Zunahme um 20,3\% je Praxisinhaber*in. Etwaige Einnahmesteigerungen bzw. kostendämpfende Entwicklungen wurden dabei weitgehend durch Mehrausgaben im Bereich Personal und IT (Wartung und Instandhaltung) aufgezehrt [23], wobei die Mehrkosten durch Coronavirus-bedingte Schutz- und Hygienemaßnahmen im Jahr 2020 noch gar nicht berücksichtigt sind.

Auch die Einführung der Datenschutzgrundverordnung (DSGVO) in den Praxen der vertragsärztlichen Versorgung war mit erheblichen Kostensteigerungen verbunden: Im Jahr 2018 mussten Praxen im Schnitt
2.487 Euro für Maßnahmen zur Umsetzung der DSGVO aufwenden. Bei Fachärzt ${ }^{*}$ innen betrug der Kostenanstieg sogar durchschnittlich 3.404 Euro in 2018 sowie 3.928 Euro in 2019. Als wesentliche Kostentreiber bei der Umsetzung der DSGVO stellten sich vor allem die Beschaffung von Software (vor allem Praxisverwaltungssysteme) und Hardware und die Aufwendungen für externes Personal wie z.B. Datenschutzbeauftragte heraus [24].

Um die flächendeckende fachärztliche Versorgung auch künftig aufrechterhalten zu können, bedarf es einer auskömmlichen Finanzierbarkeit der ambulanten Leistungen: Nicht zuletzt hat der - von der Politik vielfach aufgegriffene Begriff - des «Schutzwalls», den auch die niedergelassenen Onkolg*innen in Pandemiezeiten für die Kliniken errichtet haben, die Bedeutung einer funktionierenden ambulanten Versorgung durch Fachärzt ${ }^{*}$ innen in der Fläche für das deutsche Gesundheitssystem aufgezeigt.

\section{Ist das wirtschaftliche Risiko für privat geführte Praxen zu hoch? Referent: Andrew Ullmann, Berlin}

Trotz erheblicher Fortschritte in Diagnostik und Therapie sind maligne Tumorerkrankungen hierzulande für ein Viertel aller Todesfälle sowie maßgeblich für den Verlust an Lebensqualität verantwortlich. In der Altersgruppe der 45- bis 65-Jährigen bilden Krebserkrankungen sogar die häufigste Mortalitätsursache [25]. Die Behandlung von Krebserkrankungen gehört somit zu den großen Herausforderungen der Gegenwart, und dies nicht nur in medizinischer, sondern auch in sozioökonomischer Hinsicht.

Das pathogenetische Verständnis hat sich indessen bei vielen Tumorentitäten enorm verbessert und den $\mathrm{Zu}$ gang zu völlig neuen Behandlungskonzepten eröffnet: Neben der über viele Jahrzehnte vorherrschenden systemischen Therapie mittels unspezifisch wirksamer Zytostatika finden heute zielgerichtete medikamentöse Substanzen, Immun- und Gentherapeutika Eingang in die Patien ${ }^{*}$ innenversorgung. Obwohl es sich um ein vergleichsweise kleines Fach handelt, ermöglicht diese Bandbreite an Therapiemöglichkeiten eine zunehmend 
Table 2. Perspektiven in der Hämatologie/Onkologie: Praxis vs. Klinik/Krankenhaus

Niederlassung

Einzelchef* ${ }^{*}$ in oder Team

Work-Life-Balance: keine (kaum) Nachtschichten/Wochenenddienste

TeamWork (TU-Boards)

Faktisch nur geringe Regressrisiken

Vorerfahrungen durch Ambulanzerfahrung übertragbar

Kleines Fach

Klinische (Versorgungs-)forschung
Krankenhaus/Klinik

Chefärzt*innen-Perspektive (gemischt: Verwaltung)

Geregelte Arbeitszeiten/Urlaub

TeamWork

Kein bzw. kaum wirtschaftliches Risiko

Ambulante und stationäre Betreuung von Patient*innen Subspezialisierung

Klinische Forschung besser stratifizierte, präzisionsmedizinische Ausrichtung der onkologischen Behandlung. Angehende Fachärzt ${ }^{*}$ innen haben dementsprechend viele Möglichkeiten, sich breit zu betätigen bzw. innerhalb der Facharztausbildung auf bestimmte Tumorentitäten und Therapieansätze zu spezialisieren.

\section{Bestandsaufnahme im ambulanten Sektor}

Doch welche Perspektiven bietet die Hämatologie und Onkologie neben der hohen Innovationskraft Fachärzt ${ }^{*}$ innen, die heute vor der Frage stehen, inwieweit sich der Gang in die Niederlassung lohnen könnte? Auf der einen Seite ist eine wachsende «Ambulantisierung» zu beobachten, zumal heute viele der onkologischen Therapien auch ambulant durchgeführt werden können. Auf der anderen Seite ist der ambulante Sektor einer Reihe von strukturellen Veränderungen unterworfen: In den letzten Jahren hat die Anzahl der Medizinischen Versorgungszentren (MVZ) deutlich zugenommen, während Einzelund Gemeinschaftspraxen eher rückläufig waren bzw. in MVZ umgewandelt wurden. Gleichzeitig lässt sich eine Verschiebung in Richtung eines höheren Anteils an angestellten Hämatolog*innen und Onkolog*innen beobachten, wobei ein Angestelltenverhältnis einem späteren Einstieg als Inhaber*in der Praxis nicht entgegenstehen muss [19]. An der Trägerschaft der MVZ (alle Facharztgruppen) sind mehrheitlich Krankenhäuser (43\%) oder Vertragsärzt*innen $(41 \%)$ beteiligt (andere Investoren nur $16 \%$ ) [26].

Die Zunahme der Ärzt*innen im Angestelltenverhältnis deckt sich offenbar mit den Wünschen der angehenden Ärzt ${ }^{\star}$ innen, wobei das Interesse an einer Anstellung in einem MVZ oder in einer Praxis seit 2010 deutlicher zugenommen hat als in einem Krankenhaus. Insbesondere bei Frauen hat in diesem Zusammenhang auch der Stellenwert einer guten Work-Life-Balance zugenommen, d.h. die Vereinbarkeit von Arbeit mit Freizeit und Familie, die in der eigenen Praxis eher realisierbar scheint als im Krankenhaus [27]. Der Frauenanteil im Angestelltenverhältnis wird derzeit mit einem Drittel in den MVZ und mit ungefähr 50\% in den Praxen angegeben [19].
Ein Drittel der angehenden Ärzt ${ }^{*}$ innen kann sich derzeit den Schritt in die unternehmerische berufliche Selbstständigkeit vorstellen. Als Hauptgründe gegen den Schritt in die Selbstständigkeit werden die Angst vor drohenden Regressforderungen und die damit verbundenen finanziellen Risiken genannt - auch wenn Regresse faktisch nur selten realisiert werden. Weiterhin werden als Argumente gegen eine Niederlassung der hohe bürokratische Aufwand in den niedergelassenen Praxen und die Budgetierung aufgeführt [27]. Außerdem können externe Faktoren gegen eine eigene Praxisgründung sprechen: In Ballungsgebieten sind freie Kassensitze zum Teil schlicht Mangelware [19]. Als Pluspunkt hinsichtlich Work-LifeBalance ist aufzuführen, dass in der Onkologie und Hämatologie weder im Angestelltenverhältnis noch in der Eigenschaft als Praxisinhaber ${ }^{*}$ in mit Nachtschichten oder Wochenenddiensten zu rechnen ist (Tab. 2). Mit einem Anteil von rund 25\% leisten onkologische Schwerpunktpraxen auch einen maßgeblichen Beitrag an der Weiterbildung angehender Onkolog*innen. Zudem sind sie mit der Bearbeitung wissenschaftlicher Fragestellungen befasst: Niedergelassene Ärzt ${ }^{*}$ innen rekrutieren in einigen Bereichen bereits mehr Studienpatienten als Krankenhausärzt*innen und können auf eine leistungsfähige Infrastruktur für die klinische Forschung bzw. Versorgungsforschung vor allem im Bereich von Registerdaten zurückgreifen [28].

\section{Praxisnetzwerke als Chance}

Während Einzelpraxen auf dem Rückzug sind, nehmen Zusammenschlüsse von Hämatolog*innen und Onkolog*innen sowie ihre Einbindung in interdisziplinäre Netzwerkstrukturen zu. Der Netzwerkgedanke bietet für die Versorgung einer alternden Gesellschaft mit steigender Krebserkrankungsrate durch niedergelassene Onkolog*innen einen zukunftsweisenden Ansatz, da die Kooperation zwischen den niedergelassenen Fachärzt*innen und Leistungserbringer (z.B. im Bereich der onkologischen Rehabilitation) Verbundvorteile und Synergieeffekte schaffen kann. Der Ausbau von spezialisierten Netzwerkstrukturen zwischen Vertragsärzt*innen
Wörmann et al. 
kann neue Möglichkeiten eröffnen, flexibler auf den Wandel von Versorgungsanforderungen $\mathrm{zu}$ reagieren. Ein funktionierender Qualitätswettbewerb zum Wohle der Patient*innen bedarf allerdings homogener Ausgangsvoraussetzungen: So machen wirtschaftlich sinnvolle Kooperationsmöglichkeiten gegebenenfalls Anpassungen in der Berufsordnung der in Deutschland tätigen Ärzt*innen erforderlich (Kasten 3). Zudem gehört die kassenärztliche Bedarfsplanung auf den Prüfstand, da sie den Marktzugang reglementiert und die Etablierung von innovativen Versorgungsmodellen behindert. Neue Lösungsansätze sind auch für die unterschiedlichen Erlössituationen zwischen Krankenhausambulanz und Ambulanz in der Niederlassung gefordert: Die derzeitigen gesetzlichen Regelungentrageneherdazubei,Krankenhäuser finanziell zu bevorteilen, indem der stationäre Sektor ihnen allein überlassen ist und der ambulante Bereich zusätzlich offen steht (asymmetrischer Wettbewerb). Niedergelassene Onkolog*innen müssen überdies Wettbewerbsnachteile durch ungleiche Ausgangsvoraussetzungen bei der Finanzierung hinnehmen: Denn derzeit haben Krankenhäuser z.B. die Möglichkeit, Personal oder auch medizinisch-technisches Gerät aus dem schon refinanzierten stationären Bereich in der ambulanten Versorgung einzusetzen und eine unter Umständen defizitär betriebene Ambulanz zu subventionieren [29].

- § 12 Honorare und Vergütungsabsprachen zum Verweis auf die ärztliche Gebührenordnung,

- \$17 Niederlassung und Ausübung der Praxis, wonach es Ärzt*innen lediglich gestattet ist, über den Praxissitz hinaus an 2 weiteren Orten ärztlich tätig zu sein,

- $\$ 18$ Berufliche Kooperationen insbesondere im Hinblick auf gegebenenfalls notwendige Einschränkungen des Prinzips der freien Arztwahl

- \$31 Unerlaubte Zuweisung sowie § 33 Zuwendungen bei vertraglicher Zusammenarbeit, die in der bestehenden Fassung zu Auslegungsmissverständnissen einladen. Ökonomisch ist nicht normativ bestimmbar, wann eine bestimmte Vergütung einer bestimmten erbrachten Leistung entspricht.

Kasten 3 Diskussionswürdige Formulierungen in der bestehenden Berufsordnung für Ärzt*innen [29]
Langfristig lässt sich für die Aufrechterhaltung einer flächendeckenden Versorgung von onkologischen Patient*innen weder auf die Netzwerkbildung zwischen den niedergelassenen Fachärzt*innen noch auf die sektorenübergreifende Vernetzung mit den Zentren verzichten: Die Herausforderung wird darin bestehen, trotz zunehmender Überschneidungsbereiche die jeweiligen Kernkompetenzen herauszuarbeiten. Gleichzeitig sollte eine effizientere Versorgung nicht durch Kosten- oder Budgetierungszwänge sichergestellt werden, sondern in erster Linie über einen qualitätsbasierten Wettbewerb. Vereinfacht formuliert sollte unabhängig vom Sektor bei gleicher Qualität die gleiche Leistung auch gleichwertig honoriert werden (Kasten 4).

\section{Herausforderungen im vertragsärztlichen Bereich}

- Konkurrenz: Krankenhaus - Niederlassung (Vertragsärzt*innen)

- Unterschiedliche Bedingungen bei der Finanzierung und beim Budget

- Asymmetrischer Wettbewerb

- Personelle und finanzielle Ressourcen

\section{Optimierungsansätze}

- Ausdünnung der niedergelassenen Versorgung verhindern

- Gleiche Wettbewerbsbedingungen schaffen:

- Teilhabe an der Versorgung

- Vergütungsmöglichkeiten überdenken/faire Bezahlung ermöglichen

- Integrierte Versorgungstypen auch im ambulanten Setting umsetzen

- Homogene Netzwerke, die in den Wettbewerb treten

- Zusammenarbeit zwischen niedergelassenen Ärzt*innen und

Kliniken stärken

- Ausbau der Netzwerke (Kooperationen)

- Qualitätswettbewerb fördern

- Ärzt*innenkodex «Medizin vor Ökonomie» implementieren zum

Schutz vor dem Missbrauch ärztlicher Leistungen für ökonomisch orientierte Zwecke

Kasten 4 Herausforderungen und Optimierungsansätze im vertragsärztlichen Bereich

\section{Wie lässt sich sektorübergreifende Vernetzung und Kooperation verbindlich gestalten? Referentin: Kirsten Kappert-Gonther, Berlin}

Die Versorgung chronisch schwerstkranker Patient*innen wie in der Hämatologie und Onkologie hängt maßgeblich von der Weiterbehandlung im ambulanten Setting ab. Ein Gesundheitssystem, das durch harte Sektorengrenzen geprägt ist, erschwert eine umfassende Ver- sorgung von onkologischen Patient*innen mit hochkomplexen medizinischen Bedürfnissen. Die Trennung zwischen den Sektoren und die sektorenspezifisch ausgerichtete Vergütung sind in Deutschland historisch bedingt und die Diskussion darüber, wie die Schnittstellen zwischen den Sektoren überwunden werden können, wird bereits seit vielen Jahren als Dauerthema geführt. Doch für die Planung einer entsprechenden Behandlungskontinuität und Kooperation zwischen den Kran- 
kenhausärzt ${ }^{*}$ innen und niedergelassenen Fachärzt ${ }^{*}$ innen bedarf es vergleichbarer Ausgangsbedingungen sowohl bei der Finanzierung und Vergütung aller beteiligten Akteure als auch bei der Infrastruktur (z.B. auf digitaler Ebene), die jedoch bis heute noch nicht ausreichend auf eine sektorenübergreifende Versorgung eingestellt sind.

Kooperative Versorgungsformen wie die MVZ bieten bereits einen wichtigen Ansatz für die sektorenübergreifende Versorgung. Gerade bei den jüngeren Nachwuchsärzt*innen werden MVZ und gemeinschaftlich geführte Praxen zunehmend nachgefragt, da sie eine bessere Verteilung der Aufgaben und Lasten im Bereich der Patient*innenversorgung, Unternehmensführung und Dokumentation sowie eine flexiblere Arbeitszeitgestaltung im Sinne einer guten Work-Life-Balance ermöglichen als Einzelpraxen [19].

Die Erfahrung zeigt aber auch, dass der Gedanke von Kooperation und sektorenübergreifendem Handeln zwischen niedergelassenen Vertragsärzt* ${ }^{*}$ innen und Kliniken keinesfalls im Selbstläufermodus funktioniert: Vielmehr zeichnet sich ab, dass es deutlicher Anreize bedarf, um den Kooperations- und Vernetzungsgedanken in relevantem Ausmaß in die Praxis umzusetzen: Denn obwohl es bereits weitreichende gesetzliche Möglichkeiten für mehr Kooperation, mehr Integration und eine bessere Vernetzung gibt - zuletzt erweitert durch das Versorgungsverbesserungsgesetz (GPVG) -, besteht bis heute keine flächendeckende Umsetzung einer integrierten Versorgung und nur selten sind z.B. auch nichtärztliche Leistungserbringern in die Verträge zur integrierten Versorgung eingebunden [30].

\section{Anreize für Kooperation erforderlich}

Die Überwindung der Sektorengrenzen sowie die Schaffung von gezielten Anreizen für mehr Kooperation und Integration müssen vor diesem Hintergrund eine deutliche politische Priorisierung erfahren. Eine stärkere regionale Verankerung der Gesundheitsversorgung könnte ebenfalls zur Überwindung der sektoralen Grenzen beitragen: Ein adäquat ausgestatteter, für die Aufgaben der Vernetzung abgestellter öffentlicher Gesundheitsdienst würde bei der verbindlichen Netzwerkbildung behilflich sein und auf kommunalen oder regionalen Gesundheitskonferenzen auch die Einbindung nichtärztlicher Akteure wie Patienten- und Selbsthilfeorganisationen, Sportvereine, Bildungseinrichtungen oder Unternehmen koordinieren [31]. Werden z.B. private Kapitalinvestoren an kooperativen Versorgungsstrukturen beteiligt, dürfen allerdings potenzielle Renditeinteressen nicht die ärztliche Freiheit bei der Therapieentscheidung beeinträchtigen. Eine Stärkung der Position der Ärztlichen Leiter*innen im MVZ ist auch vor diesem Hintergrund ein wichtiger Schritt. Im Interesse der versicherten Patient*innen muss die gleiche Transparenz über die
Trägerstrukturen eingefordert werden, wie sie heute zunehmend auch von den Leistungserbringern abverlangt wird.

In Anlehnung an den $\$ 140$ a SGB V («Besondere Versorgungsformen») hat die Fraktion Bündnis 90/Die Grünen im August 2020 den Antrag «Gesundheitsregionen - Aufbruch für mehr Verlässlichkeit, Kooperation und regionale Verankerung in unserer Gesundheitsversorgung» eingebracht. Er enthält Vorschläge zur Regelung von sektoren- und indikationenübergreifenden, populationsorientierten und regionalen Versorgungsverträgen: Die gesetzlichen Krankenkassen würden damit die Möglichkeit haben, sogenannte "Gesundheitsregionenverträge» mit regionalen Gesundheitsverbünden abzuschließen. Als geeigneter Bezugsrahmen für die «Gesundheitsregionen» würden z.B. die Gebiete von Landkreisen oder anderen Gebietskörperschaften mit etwa 150.000 Einwohner*innen in ländlichen Räumen erachtet. Gesundheitsverbünde können insbesondere Zusammenschlüsse regionaler Leistungserbringern (z.B. Ärzt ${ }^{*}$ innennetze, Qualitätsnetze) sein. Neben schon bestehenden Aktivitäten würden auch Verbindungen von sozialen Einrichtungen, Patient*innenorganisationen, sozialpsychiatrischen Einrichtungen, Krankenhäusern und weiteren Akteuren beteiligt. Die Organisation der ambulanten und stationären Versorgung sowie die virtuelle Budgetverantwortung würde der Gesundheitsverbund als Vertragspartner der Krankenkassen übernehmen. Die Vergütung würde auf der Grundlage der bestehenden Vergütungssysteme erfolgen oder auch auf eigenen Vergütungsregelungen für den ambulanten Bereich in der Region beruhen, die Spielraum für andere/ zusätzliche Anreize setzen und gegebenenfalls auch ohne Budgetierungen auskommen. Auf Basis einer gesetzlichen Regelung würden - als finanzieller Anreiz - Krankenkassen, die einen Gesundheitsregionenvertrag abgeschlossen haben, für einen Zeitraum von 10 Jahren einen Zuschlag auf die Zuweisung für jeden ihrer in dieser Region lebenden Versicherten erhalten [31].

\section{Neuordnung der Arbeitsverteilung im}

Gesundheitswesen

Um den regional unterschiedlichen Anforderungen für eine verbesserte Gesundheitsförderung gerecht $\mathrm{zu}$ werden und mehr Fachärzt*innen für eine Tätigkeit in strukturschwachen Regionen zu gewinnen, werden auch praktische Aufgaben bewältigt werden müssen, wie z.B. die verbesserte Anbindung von Gesundheitseinrichtungen an den öffentlichen Nahverkehr oder die Vorhaltung einer geeigneten Infrastruktur für telemedizinische Angebote und die digitale Verknüpfung der Sektoren.

Chronisch kranken Tumorpatient ${ }^{*}$ innen kann in einem weniger von Sektorengrenzen geprägten Gesundheitswesen eine umfassendere, teambasierte Versorgung 
zuteilwerden: So könnten z.B. speziell ausgebildete Pflegefachpersonen eine stärkere Rolle übernehmen und die Public-Health-Perspektive würde im Sinne von Gesundheitsprävention und -förderung einen höheren Stellenwert erhalten. Dazu kann es notwendig sein, überholte Aufgabenverteilungen in der Gesundheitsversorgung anzupassen und die Arbeitsverteilung zwischen ärztlichen und nichtärztlichen Gesundheitsberufen einer Neuordnung zu unterziehen (Kasten 5) [31].
Ausschnitt aus dem Antrag der Fraktion Bündnis 90/Die Grünen zur sektorenübergreifenden Versorgung

- Schaffung einer gesetzlichen Regelung für «Gesundheitsregionen» zu sektoren- und indikationsübergreifenden, populationsorientierten und regionalen Versorgungsverträgen («Gesundheitsregionenverträge»), die von den gesetzlichen Krankenkassen mit regionalen Gesundheitsverbünden abgeschlossen werden

- Gezielte Anreize setzen: Gesetzlichen Krankenkassen, die für ihre Versicherten einen solchen Gesundheitsregionenvertrag abgeschlossen haben oder einem solchen Vertrag beigetreten sind, wird für die Dauer von 10 Jahren eine erhöhte Zuweisung aus dem Gesundheitsfonds für ihre im Geltungsbereich des Gesundheitsregionenvertrages lebenden Versicherten gewährt

- Verschiedene Institutionen (z.B. Aufsichten über die gesetzlichen Krankenkassen, regionale Gesundheitskonferenzen) erhalten ein Initiativrecht, das es ihnen ermöglicht, Krankenkassen zum Abschluss von Gesundheitsregionenverträgen aufzufordern; Krankenkassen, die keine derartigen Verträge abschließen, unterliegen einer Begründungspflicht

- Das Institut für Qualitätssicherung und Transparenz im Gesundheitswesen (IQTIG) wird mit der Evaluation von Gesundheitsregionen beauftragt

- Zielmarke: 10\% der Versicherten bis 2025 in integrierten Versorgungsverträgen versorgen

Kasten 5 «Gesundheitsregionen - Aufbruch für mehr Verlässlichkeit, Kooperation und regionale Verankerung in unserer Gesundheitsversorgung»; mod. nach [31].

\section{Stationäre Versorgung Wer entscheidet: Ärzteschaft oder Controlling? Referent: Hermann Einsele, Würzburg}

Das Patient ${ }^{*}$ innenwohl gilt als normativer Maßstab der Gesundheitsversorgung. Die Wahrung des $\mathrm{Pa}$ tient*innenwohls hängt dabei nicht nur von der körperlichen und seelischen Situation nach medizinischen Maßstäben ab. Auch die Behandlungsqualität, die Selbstbestimmung der Patient ${ }^{\star}$ innen sowie die Zugangs- und Verteilungsgerechtigkeit bei knappen Ressourcen sind Bestandteil eines ethischen Orientierungsrahmens im stationären Versorgungssystem. Durch die wachsende Fokussierung auf eine Reduktion der Ausgaben aufseiten der Krankenkassen und Ertragssteigerung aufseiten der Anbieter entstehen jedoch Effekte, die das $\mathrm{Pa}$ tient*innenwohl als normative Funktion gefährden [32].

\section{Ökonomisierung versus Berufsethik?}

Zwar befinden sich Ärzt*innen, wie in jedem anderen Beruf auch, in einem Spannungsfeld zwischen berufsethischen und ökonomischen Interessen. Ökonomische Anreizsysteme in der Medizin erzeugen allerdings Druck, der dazu führen kann, dass der Vorrang der medizinischen Ethik und damit der ärztlichen Entscheidungsund Therapiefreiheit abnimmt [33].

Während Wirtschaftlichkeit in der Medizin einen klugen Umgang mit Ressourcen, Prozessoptimierung und Vermeidung von Verschwendung umfassen sollte, kommt es zu ei- ner übertriebenen Ökonomisierung, wenn Fehlanreize gesetzt werden, um Gewinn zu maximieren (z.B. durch Fallzahlsteigerung oder Priorisierung von teuren Fällen). Der Einzug von Privatisierung, Wettbewerb und Gewinnstreben sind an sich nicht negativ, sofern sie eine Qualitätssteigerung und Fortschritt ermöglichen. Allerdings lässt sich die damit verbundene Tendenz beobachten, dass Überlegungen zur Erlössteigerung und ökonomische Ziele in den Vordergrund treten und berufsethische Leitgedanken ihren primären Stellenwert verlieren [33]. Als Beispiele sind Zielvereinbarungen von leitenden Ärzt*innen aufzuführen, die Operationszahlen mit jährlichen Untergrenzen vorsehen oder fallzahlabhängige Boni in Ärzt*innenverträgen, die zu ökonomisch begründeten Indikationsstellungen verleiten.

Sinnvoll ist eine Ökonomisierung der Kliniken im Sinne eines optimierten medizinischen Prozessmanagements, wenn berufsethische Grenzen nicht überschritten und die Unterschiede zum kaufmännischen Denken in der freien Wirtschaft berücksichtigt werden (Kasten 6) [33].

\footnotetext{
- Steigerung des Patient*innennutzens und der Wertschöpfung im Gesundheitssystem

- Leistungsbezogene Honorierung bei rein medizinisch begründeter Indikation

- Prozessoptimierung mit Vermeidung von Verschwendung

- Voraussetzung: ethische Abwägung bei allen gesetzten Anreizen zum Schutz vor Fehlanreizen
}

Kasten 6 Ziele des medizinischen Prozessmanagements und der Wirtschaftlichkeit; mod. nach [33]. 
Table 3. Positive und negative Folgen der Ökonomisierung; mod. nach [33].

\begin{tabular}{|c|c|}
\hline Positive Folgen der Ökonomisierung & Negative Folgen der Ökonomisierung \\
\hline $\begin{array}{l}\text { - Patient*innenorientierte Versorgungsformen } \\
\text { - Kontinuierliche Aktualisierung der Leitlinien sowie der kliniktypischen } \\
\text { Behandlungsabläufe } \\
\text { - Hinterfragen jeder Routinemaßnahme auf ihre Notwendigkeit } \\
\text { - Überprüfung diagnostischer Abläufe in festen Intervallen hinsichtlich } \\
\text { potenzieller Modernisierung } \\
\text { - Befragung ärztlicher Zuweiser*innen und Leiter }{ }^{*} \text { innen von Übernahme- } \\
\text { Kliniken zum eigenen Reformbedarf }\end{array}$ & $\begin{array}{l}\text { - Rückgang von Qualität durch DRGs in der Wahrnehmung der } \\
\text { Krankenhausärztärzt*innen (49\%) und Pflegekräfte (37\%) } \\
\text { - Zunehmender Druck auf die «Produktivität» der Ärzt*innen (Fallzahlen, } \\
\text { «lukrative» Eingriffe) } \\
\text { - Finanzielle Anreize, die zulasten der sprechenden Medizin gehen } \\
\text { - Statt ärztlicher Diskussion von Diagnose/Differenzialdiagnosen } \\
\text { Überlegungen zu ökonomisch lohnender DRG-Verschlüsselung und Case- } \\
\text { Mix-Index } \\
\text { - Verlust des Mehraugenprinzips durch Trend zu «Ambulantisierung» } \\
\text { - Ökonomische Überreizung führt zu höheren Krankenständen, personeller } \\
\text { Fluktuation, innerer Emigration des Personals } \\
\text { - Zunehmende Demotivierung der Ärzt*innen, wenn Regeln der } \\
\text { Marktwirtschaft zur Basis des ärztlichen Handelns werden }\end{array}$ \\
\hline
\end{tabular}

\section{Vorrang des Patient*innenwohlergehens}

Neben der Problematik (finanzieller) Fehlanreize als negative Folge der Ökonomisierung nehmen Ärzt ${ }^{*}$ innen und Pflegekräfte mehrheitlich eine Verschlechterung der Qualität wahr, die der Einführung des DRG-Systems von 2003 zugeschrieben wird [33] und der damit einhergehenden Verschärfung des Kostendrucks. Die Einführung der diagnosebezogenen Fallpauschalen (diagnosis-related groups, DRGs) hatte ursprünglich eine optimierte Prozess-, Struktur- und Ergebnisqualität der ärztlichen Diagnostik sowie die verbesserte Nutzung von Ressourcen in der stationären Versorgung zum Ziel [32]. Allerdings steigen die Kosten für die Eingriffe schneller an als die Fallpauschalen, sodass den Krankenhäusern am Ende eines Jahrs weniger Geld pro Intervention verbleibt. Infolgedessen werden höhere Fallzahlen und lukrativere Eingriffe (z.B. an der Wirbelsäule, Herzkatheteruntersuchungen) angestrebt [34]. Auch bilden die derzeitigen Vergütungsund Erlössysteme die «sprechende Medizin» nicht hinreichend $\mathrm{ab}$, während technische Leistungen und kurze Verweildauern «belohnt» werden [33]. Wirtschaftliche Überlegungen rücken auch die Maximierung des CaseMix-Indexes (durchschnittliche Erkrankungsschwere) in den Vordergrund, während eine ärztlich geführte Diskussion über die Richtigkeit einer Diagnose und mögliche Differenzialdiagnosen in den Hintergrund gerät $[33,35]$. Des Weiteren geht die zunehmende «Ambulantisierung» einer ehemals stationären Diagnostik mit dem wachsenden Verlust des Mehraugenprinzips einher (Tab. 3). Für die Behandler*innen ist es demotivierend, wenn ärztliches Handeln zunehmend den Prinzipien der Marktwirtschaft unterworfen und eine von Respekt und authentischer Fürsorge geprägte Beziehung zwischen Ärztin und Patient ${ }^{\star}$ in erschwert wird [33].

Patient ${ }^{*}$ innen sollten aber darauf vertrauen können, dass ihr Wohlergehen Vorrang vor ökonomischen Überlegungen hat $[33,36]$. Daraus geht hervor, dass die Ziele der Marktwirtschaft nicht mit den Zielen der Medizin gleichgesetzt werden dürfen: Die Ökonomie muss die Rahmenbedingungen für ärztliches Handeln optimieren, darf jedoch ärztliches Handeln nicht bestimmen. Denn das Wohlergehen der Patient*innen hat immer Vorrang vor wirtschaftlichen Überlegungen - sowohl aus berufsethischen als auch aus rechtlichen Gründen (Genfer Gelöbnis des Weltärztebundes) [33].

\section{Ökonomisierung patient*innenbezogener \\ Entscheidungen}

Die Sicht der Krankenhausakteure wurde in einer qualitativen, interviewbasierten Studie mit der Zielsetzung untersucht, mögliche Zusammenhänge zwischen den finanziellen Rahmenbedingungen der Krankenhäuser, dem betriebswirtschaftlichen Management und ärztlichen patient*innenbezogenen Entscheidungen zu beleuchten. Die Erhebung fand zwischen 2013 und 2016 statt, in der eine repräsentative Stichprobe von bundesweit 31 Geschäftsführer*innen und 32 Ärzt $^{*}$ innen ausführlich in Pilot- und Leitfadeninterviews befragt wurde $[35,37]$. Ergänzend wurden Fokusgruppen, Gruppengespräche, schriftliche Befragungen und ein Werkstattgespräch durchgeführt.

Bei der Wahrnehmung medizinischer und ökonomischer Prozesse zeichneten sich deutliche Unterschiede zwischen Befragten in der Geschäftsführung versus der Ärzteschaft ab: Während Geschäftsführer*innen ausnahmslos verneinten, ärztliche Entscheidungen zu beeinflussen, beschrieben die befragten Ärzt*innen deren Einflussnahme auf medizinische Entscheidungen als eine nahezu alltägliche Tatsache [37]. Fast einmütig berichteten Ärzt ${ }^{*}$ innen, dass viele der betriebswirtschaftlichen Vorgaben ärztliche Entscheidungen direkt oder indirekt beeinflussten und sie sich mehr oder weniger dazu gezwungen sehen würden, Einflussnahmen auf die Behandlung zu akzeptieren, oder dass sie sich von sich aus wirtschaftlichen Zwängen gebeugt hätten. Aus ärztlicher Perspektive zu den stärksten indirekten Einflussnahmen gehörten 
die Bemessung der Personalstärke und die Entscheidungen über die Eröffnung oder Schließung von Abteilungen und Stationen durch die Geschäftsführung. Weiterhin wurde auch die Material- und Medikamentenbeschaffung als indirekte Einflussnahme auf die medizinische Praxis empfunden. Die befragten Ärzt ${ }^{\star}$ innen berichteten zudem über versuchte oder praktizierte direkte Einflussnahmen, «wenn es um Fallzahlen oder den Case-Mix-Index geht» [35].

Als Beispiele für betriebswirtschaftlich motivierte Aufforderungen an die Ärzteschaft wurden unter anderem die Maximierung der Belegung, Vermeidung aufwendiger «unlukrativer» Patient*innen, Ausrichtung der Verweildauer nach DRGs oder Auslastung gewinnbringender Geräte und Prozeduren benannt (Kasten 7). Invasive und apparative Prozeduren wurden konservativen Verfahren vorgezogen, abwartende und sprechende Medizin wirtschaftlich bestraft. Viele Ärzt*innen sahen die Grundsätze medizinischer Ethik verletzt [35]. Nahezu alle befragten Ärzt ${ }^{*}$ innen berichteten Arbeitsverdichtung, Zeitknappheit und Stress. Dies wiederum wurde mit Auswirkungen auf die Qualität der Entscheidungen und Risiken für Personal und Patient*innen in Verbindung gebracht [35].

\section{Beispiele betriebswirtschaftlich motivierter Aufforder- ungen an die Ärzteschaft \\ - Maximierung der Belegung (auch unter Inkaufnahme höherer Risiken für Patient*innen) \\ - Steigerung der Fallzahlen \\ - «Unlukrative» aufwändige Patient*innen möglichst vermeiden \\ - Notaufnahmen bei Überlastung nicht beim Rettungsdienst als Anlaufstelle abmelden \\ - Ausrichtung der Verweildauer nach DRGs \\ - Maximierung des Case-Mix-Index \\ - «Kreative Indikationen» stellen (unnötige Behandlungen) \\ - Auslastung gewinnbringender Geräte, Prozeduren und Einrich- tungen (OP, Endoskopie, Intensiveinheiten, Beatmungsplätze, Spezialdiagnostik, Herzkatheter) \\ - «Fallsplitting» (Entlassung und Wiederaufnahme von \\ Patient*innen statt hausinterne Verlegung) \\ - Patient*innen an die Klinik binden, auch wenn sie andernorts besser behandelt würden \\ - Bevorzugte Versorgung von Privatpatient*innen}

Kasten 7 Einfluss der Ökonomisierung auf Aufnahme, Behandlung und Entlassung (mod. nach [35])

Als zentralen Ausgangspunkt der Einflussnahme identifizierte die Mehrheit der befragten Ärzt*innen die Geschäftsführung, die Verantwortlichen des Trägers oder den Konzernvorstand [35]. So läuft es an vielen deutschen Kliniken auf eine monatliche, teilweise wöchentliche Konfrontation mit Kennzahlen durch die Klinikleitung hinaus, die einen enormen Druck auf die Chefärzt ${ }^{*}$ innen erzeugen, der wiederum auf die Oberärzt ${ }^{*}$ innen übertragen wird. Eine Krankenhaus-Controlling-Studie ergab für die
Jahre 2014-2015, dass rund drei Viertel der Krankenhäuser mit den Ärzt*innen Leistungsziele, wie für bestimmte Umsätze und operative Eingriffe, vereinbart haben [34].

Aus der Sicht der Geschäftsführung besteht die Notwendigkeit, eine ausreichend hohe Rendite zu erwirtschaften, über die sich die Zukunft der Institutionen sichern lässt. Es wurde betont, dass auf Einzelfallentscheidungen im Behandlungsprozess kein Einfluss genommen werde und die Geschäftsführung nicht wünscht, dass Patient ${ }^{*}$ innen ohne medizinische Indikation stationär aufgenommen würden. Bei der Betrachtung der Antworten von Ärzt*innen und Geschäftsführer*innen auf ausgewählte Fragen im Leitfadeninterview fiel allerdings auf, dass die Einschätzungen beider Gruppen mehrheitlich diskrepant waren: Beispielsweise bejahten 9 von 20 befragten Ärzt*innen die Frage, ob aus wirtschaftlichen Motiven für Patient ${ }^{\star}$ innen gesondert abrechenbare Leistungen erbracht werden (z.B. Herzkatheteruntersuchung), wohingegen die Mehrheit der Geschäftsführer*innen die Frage verneinte (17 von 21 ). Die Frage, ob medizinische Abteilungen und Verfahren, die Einnahmen generieren, bevorzugt ausgebaut würden (auch wenn sie nicht immer dem medizinischen Bedarf entsprechen), wurde von 18 der 20 befragten Ärzt*innen und von 13 der 21 befragten Geschäftsführer*innen verneint [35].

\section{Medizin-Controller*innen als Vermittelnde?}

Die Mehrheit der Krankenhäuser ist noch auf der Suche nach dem adäquaten Management: Über 70\% der Kliniken befanden sich einer Studie zufolge 2015 in einer Restrukturierungsphase, über die Hälfte wechselte zumindest einen Teil ihrer Geschäftsführung aus - im Unterschied zu den leitenden Ärzt*innen, die in der Regel 20 Jahre und länger auf ihrer Position verbleiben [38]. Eine intensivierte $\mathrm{Zu}$ sammenarbeit und Verständigung zwischen Ärzteschaft und Geschäftsführung über die Verteilung der Mittel erscheint unumgänglich. Dazu gehört aufseiten der Ärzt*innen die Auseinandersetzung mit ökonomischen Gesetzmäßigkeiten ebenso wie das Einbringen von eigenen Gestaltungsvorschlägen und das Ansprechen von problematischen Entwicklungen [37]. Eine Vermittlerfunktion an der Schnittstelle von Krankenhausalltag, Geschäftsführung und Ärzt*innen übernehmen heute Medizin-Controller, indem sie die betriebswirtschaftlichen Konsequenzen medizinischer Prozesse verdeutlichen: Das verbesserte Verständnis der Handlungsentscheidungen des Managements bzw. des Controllings soll eine optimierte Zusammenarbeit aller Akteure und im Idealfall den Chefärzt ${ }^{*}$ innen gemeinsam mit der Geschäftsführung gute strategische Entscheidungen ermöglichen. Beim Zugang zu den wirklich relevanten Daten, wie z.B. die Ergebnisse einer Deckungsbeitragsrechnung und einer Einzelfallerlösberechnung, besteht jedoch häufig noch ein Ungleichgewicht und die Informationen, die die Chefärzt ${ }^{*}$ innen erreichen, 
werden durch die Geschäftsführung gefiltert [38]. Damit Controlling und leitende Ärzt ${ }^{*}$ innen in diesem Spannungsfeld nicht länger gegeneinander arbeiten, sollten prinzipiell auch Medizin-Controller*innen über medizinische Grund- kenntnisse verfügen und sich ein eigenes Bild über den Klinikalltag machen. Zudem erscheint es aussichtsreicher, das bestehende System vermehrt auf Transparenz und Qualität der medizinischen Leistung auszurichten [38].

\section{Stationäre Versorgung in der Onkologie - Risiko für Fehlanreize? Referent: Martin Siess, München}

Bei der Frage, wo die größten Risiken für Fehlanreize in derstationären VersorgungonkologischerPatient ${ }^{\star}$ innen bestehen, richtet sich der Blick unweigerlich auf die Indikationsstellung: insbesondere für Therapien, aber auch im Bereich der invasiven Diagnostik. Die Indikationsstellung ist allerdings auch aus Sicht der onkologischen $\mathrm{Pa}$ tient ${ }^{\star}$ innen und in Bezug auf die Ergebnisqualität von entscheidender Bedeutung, wobei Wunschbild und Realität häufig auseinandergehen: Dem Wunschbild würde entsprechen, dass die Indikationsstellung gemeinsam mit dem Betroffenen (und gegebenenfalls den Angehörigen) ausschließlich nach Maßgabe medizinischer, wissenschaftlicher, ethischer und patient*innenindividueller Aspekte erfolgt. In der Realität aber lässt sich ein zunehmender Einfluss wirtschaftlicher Faktoren auf die Indikationsstellung feststellen, die von den Kosten der Behandlung über die Refinanzierung bis hin zu ökonomischen Zwängen auf Klinik, Abteilung und die behandelnden Ärzt*innen reichen. Fehlanreize und Schieflagen in der onkologischen Krankenhausversorgung resultieren durch problematische Aspekte des Klinikmanagements (interne Budgetierung), der Chefärzt ${ }^{*}$ innenverträge/Zielvereinbarungen (vgl. auch Beitrag H. Einsele), der Struktur des Gesundheits- und Krankenhauswesens sowie der Vergütungsmodelle und Refinanzierung.

\section{Strukturelle Probleme im Gesundheits- und \\ Krankenhauswesen}

Das widersprüchliche Nebeneinander von Wettbewerb/Konkurrenz und bedarfsorientierter Versorgung ist eine Besonderheit des gegenwärtigen Krankenhauswesens in Deutschland. Die Einführung des fallpauschalierten Vergütungssystems (DRG-System) vor 18 Jahren hat zwar zur (erwünschten) Zunahme des Wettbewerbs zwischen den Kliniken geführt. Gleichzeitig nahm aber die Ausgabenentwicklung trotz der vermehrten Transparenz über erbrachte Leistungen und die resultierenden Kosten häufig nicht ab [39]. Nach den DRGs werden Krankenhausleistungen aber nicht nach den jeweiligen Ist-Kosten vergütet, sondern nach festgelegten Soll-Kosten für unterschiedliche Fallgruppen. Dadurch können Fehlanreize wie z.B. der überproportionale Anreiz zur
Fallzahlsteigerung oder zur Anpassung des Case-MixIndex an die ertragsträchtigsten DRGs entstehen, die Unterversorgung und Patient*innenselektion begünstigen (wenn die Kosten höher sind als die Fallpauschale) bzw. Über- und Fehlversorgung (wenn die Kosten niedriger sind als die Fallpauschale).

Liegen die Kosten für eine Fallpauschale oberhalb der Vergütung, werden Anstrengungen unternommen, Kosten zu senken. Dabei werden auch Anreize zu einem reduzierten Einsatz medizinisch-technischer Innovationen gesetzt. Verglichen mit den Anreizen, Kosten zu minimieren, fallen die DRG-System-immanenten Qualitätsanreize deutlich schwächer aus [39]. Zusätzlich erhöht sich der wirtschaftliche Druck auf die Krankenhäuser über die Budgetierung. Überdies ziehen sich die öffentlichen Träger zunehmend aus der wirtschaftlichen Verantwortung für ihre Krankenhäuser zurück [40]. Derzeit nehmen Übernahmen durch Private-Equity-Gesellschaften im ambulanten Sektor der Onkologie an Fahrt auf [41] - mit besorgniserregenden zusätzlichen Risiken für die bedarfsgerechte Patient*innenversorgung.

Nach zahlreichen Reformen weist das DRG-System mittlerweile eine hohe Komplexität sowie einen hohen Bürokratisierungsgrad auf und die bisherigen gesundheitspolitischen Versuche, die onkologische Versorgung sektorenübergreifend weiterzuentwickeln, haben Fehlanreize nicht verhindern können. Tatsächlich schneidet Deutschland im internationalen Vergleich bei der Koordination von Primär- und Krankenhausversorgung schlechter ab als andere Länder: Im Durchschnitt sucht hierzulande nur etwa die Hälfte der onkologischen Patient ${ }^{*}$ innen eine onkologische Facharztpraxis auf [42].

\section{Fokus auf mehr Versorgungsorientierung}

Für eine mehr bedarfsgerechte Versorgung sollten Krankenhäuser noch stärker im Hinblick auf Strukturqualitätskriterien gefördert werden und nicht angereizt sein, bestimmte medizinische Leistungen zur Erlösoptimierung vermehrt durchzuführen bzw. ausgesuchte Patient ${ }^{*}$ innenkollektive eher zu behandeln. Ein hoher Stellenwert ist vor diesem Hintergrund den Initiativen der Fachgesellschaften sowie den Aktivitäten im Rahmen des Nationalen Krebsplans (NKP) beizumessen, der vom Bundesministerium für Gesundheit (BMG) gemeinsam mit der Deutschen Krebsgesellschaft (DKG), der Deutschen Krebshilfe (DKH) und der Arbeitsgemeinschaft 
Abb. 12. Quellen für Fehlanreize: Struktur des Gesundheits- und Krankenhauswesens.

\section{Themen}

- Nebeneinander von Wettbewerb und Bedarfsorientierung im Krankenhauswesen

- Fortlaufend neue Gesundheitsreformen und Regulierungsversuche (z.B. G-BA) mit erheblicher Ausweitung der Bürokratie

- Versuche der Gesundheitspolitik zur Weiterentwicklung der onkologischen Versorgung (ambulant-stationär)

- Wichtige Initiativen durch Fachgesellschaften (z.B. DKG, DGHO), Krebshilfe und BMG (Nationaler Krebsplan)
Auswirkungen / Fehlentwicklungen

- Fehlanreize führen zu Über-, Unterund Fehlversorgung in der Onkologie

- Innovationshemmnisse in der Onkologie neben Leistungsausweitung durch Wettbewerb

- Nur 50\% derPatient*innen befinden sich zur Primärversorgung in ausgewiesenen onkologischen Strukturen

- Qualität/Ergebnisqualität der onkologischen Versorgung hat immer noch zu geringe Steuerungswirkung

- Private Equity dringt zunehmend in ambulante Onkologie vor
Abb. 13. Quellen für Fehlanreize: Vergütungsmodelle und Refinanzierung.

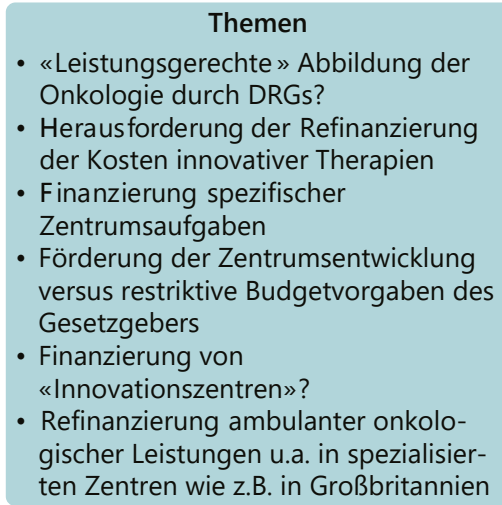

Themen

- «Leistungsgerechte» Abbildung der Onkologie durch DRGs?

- Herausforderung der Refinanzierung der Kosten innovativer Therapien

- Finanzierung spezifischer Zentrumsaufgaben

- Förderung der Zentrumsentwicklung versus restriktive Budgetvorgaben des Gesetzgebers

- Finanzierung von «Innovationszentren»?

- Refinanzierung ambulanter onkologischer Leistungen u.a. in spezialisierten Zentren wie z.B. in Großbritannien

\section{Auswirkungen / Fehlentwicklungen}

- Onkologische Leistungen mit hoher Fallschwere/wichtige Erlösquelle für Kliniken

- Innovative Therapien und onkologische Sachkosten häufig nicht kostendeckend

- Hochwertige Zentrumsstrukturen und -aufgaben nicht ausreichend gegenfinaziert

- Zentrumsbildung gegen die Budgetregelungen des Gesetzgebers/ gegen die Interessen der Kassen

\section{Maßnahmen gegen Fehlanreize}

- Koppelung der Finanzierung an Strukturvorgaben für die onkologische Versorgung/ - Koppelung
Deutscher Tumorzentren initiiert wurde, um Problembereiche in der Tumorfrüherkennung und -versorgung $\mathrm{zu}$ adressieren und weiterzuentwickeln [43]. Die DKH beispielweise fördert seit 2007 onkologische Spitzenzentren, die neben der qualitätsgesicherten Versorgung auch übergreifende Forschungs-, Entwicklungs- und Vernetzungsaufgaben übernehmen. Durch die Umsetzung von Onkologie-Konzepten, die auf Qualitätsförderung sowie interdisziplinäres und sektorenübergreifendes Innovationsmanagement setzen, bieten sich Chancen, eine bessere Versorgungsorientierung zu erreichen und strukturell bedingten Fehlanreizen entgegenzusteuern (Abb. 12). Bei der Ausgestaltung und Umsetzung ist die verstärkte Einbindung von Gesundheitspolitik, des G-BA, der Kostenträger und Länder anzustreben.

\section{Vergütungsmodelle und Refinanzierung}

Das Problem der Fehlanreize ist systemimmanent und wird im Grundsatz nicht lösbar sein, solange die DRGPauschalen in Deutschland als alleinige Abrechnungsgrundlage dienen (Abb. 13). So stellen onkologische Leistungen mit hoher Fallschwere für die Krankenhäuser eine wichtige Erlösquelle dar. Mehrleistungen, wie sie in den Krebszentren erbracht werden, lassen sich durch ein bud- 
getiertes Vergütungssystem mit Mehrleistungsabschlägen aber nicht vollständig gegenfinanzieren.

Darüber hinaus weisen innovative onkologische Therapien eine sehr hohe Kostenrelevanz auf und werden in der derzeitigen Versorgungskette häufig nur inadäquat abgebildet.

Bei der Überführung innovativer Verfahren der Onkologie in den klinischen Alltag kommt ausgewählten Spitzenzentren an universitären Standorten (Comprehensive Cancer Centers, CCCs) eine wichtige Rolle zu, da sie die Erprobung der Therapien unter kontrollierten Bedingungen des Versorgungsalltags ermöglichen und zum Erhalt eines bundesweit hohen Versorgungsniveaus onkologischer Patient*innen beitragen. Spezifische Strukturkomponenten und Mehrleistungen wie z.B. Tumorkonferen- zen, Zweitmeinungen, regionale Koordination und Patient*innensteuerung sowie Register und Tumor-/Biobanken, die an den CCCs erbracht werden, verursachen allerdings höhere Kosten sowie Mehraufwand durch die höhere Komplexität der Leistungen. Zentrumszuschläge ermöglichen derzeit noch keine flächendeckend auskömmliche Finanzierung der onkologischen Spitzenzentren. Und auch die spendenfinanzierte Deutsche Krebshilfe wird die notwendigen Strukturkomponenten der Krebszentren auf Dauer nicht fördern können. Zur Finanzierung von Spitzenzentren und Innovation sind folglich neue kostendeckende Ansätze gefragt, wie z.B. in Großbritannien oder den USA, die Nationale Krebszentren mit staatlichen Investitionen ausstatten, um Spitzenforschung und -versorgung sicherzustellen $[44,45]$.

\section{Was kostet gute Pflege - oder was ist uns gute Pflege wert? \\ Referent: Christian Wylegalla, Freiburg}

Die Situation in den Pflegeberufen gehört zu den gegenwärtigen großen Herausforderungen für Gesellschaft und Politik: Mit aktuell etwa 900.000 Frauen und Männern, die über eine mindestens 3-jährige pflegerische Ausbildung verfügen, repräsentiert das Pflegepersonal die mit Abstand größte Berufsgruppe im deutschen Gesundheitswesen. Im Zuge der mit der demografischen Entwicklung einhergehenden Zunahme multimorbider Patient*innen sowie komplexerer Krankheitsbilder zeichnet sich der steigende Pflegebedarf bereits seit vielen Jahren ab [46]. Auf der anderen Seite ist auch der Fachkräftemangel demografisch geprägt und stellt das Personalwesen im Gesundheitsbereich vor weitere enorme Herausforderungen. Allein in der stationären onkologischen Krankenversorgung fehlen bereits heute 70.000 Pflegekräfte [47]. Auch wenn die Arbeitsbedingungen und der Fachkräftemangel in der Pflege zuletzt auch vermehrt in der medialen Öffentlichkeit diskutiert und in die politische Agenda aufgenommenen wurden, wurde der Pflegedienst in der Vergangenheit vor allem als Kostenfaktor betrachtet: Verglichen mit anderen Berufsgruppen im Gesundheitswesen war die Pflege oftmals besonders stark vom Personalabbau betroffen [48]. Während der COVID-19-Pandemie hat die Situation der Intensivpflegekräfte erneut ein Schlaglicht auf die Arbeitsbedingungen und die Attraktivität des Berufsstands geworfen - übertragbar auf die Pflege insgesamt und charakterisiert durch eine zu große Arbeitsbelastung, inadäquate Vergütung und zu wenig Zeit für Patient*innen. Einen dringenden Verbesserungsbedarf konstatieren die Deutsche Gesellschaft für Fachkrankenpflege und Funktionsdienste e.V. (DGF) und die Deutsche interdisziplinäre Vereinigung für Intensiv- und Notfallme- dizin (DIVI) in ihrer aktuellen Stellungnahme nicht nur für die Arbeitsbedingungen, sondern auch für die Personalfürsorge, interprofessionelle Teamarbeit, Weiterbildung und Schaffung beruflicher Perspektiven $[49,50]$.

\section{Ergebnisforschung in der Pflege}

Wie systemrelevant ausreichend eingesetztes und gut ausgebildetes Pflegefachpersonal für die Patient*innensicherheit und Versorgungsqualität ist, verdeutlichen die Ergebnisse der bislang größten, europaweit durchgeführten Pflegestudie RN4CAST (Nurse Forecasting: Human Resources Planning in Nursing). In der Querschnittsstudie wurden zwischen 2009 und 2010 insgesamt 33.659 Pflegekräfte in 12 EU-Ländern an 488 Akutkrankenhäusern zu ihrem Arbeitsalltag befragt [51]. Bis auf 3 Ausnahmen (Deutschland, Polen, Griechenland) waren in 9 Ländern auch Daten zur postoperativen Patientenmortalität verfügbar: Demnach nahm die Wahrscheinlichkeit, dass Patient ${ }^{\star}$ innen nach einem operativen Eingriff stationär versterben, mit steigender Arbeitsbelastung der Pflegekräfte zu. Zudem bewirkte eine Steigerung des Anteils von Pflegenden mit Bachelor-Abschluss um 10\% eine Verringerung der Mortalitätsrate um 7\% [52].

Im deutschen Teil der RN4CAST-Studie, an der 49 Akutkrankenhäuser beteiligt waren, wies keine einzige Pflegefachkraft einen Bachelor-Abschluss auf [51, 52]. Einer Empfehlung des Wissenschaftsrats zufolge wäre es sinnvoll, 10-20\% eines Ausbildungsjahrgangs in den Gesundheitsfachberufen akademisch zu qualifizieren [53]. Ein weiterer Aspekt, der Deutschland gegenüber anderen Ländern ungünstig abschneiden lässt, betrifft die Stellenbesetzung und die Patienten-zu-Pflegepersonal-Relation: Mit 13,0 durchschnittlich zu betreuenden Patient*innen pro Pflegefachkraft liegt Deutschland nach Spanien $(12,6)$ und Belgien $(10,7)$ an letzter Stelle (Tab. 4). 
Table 4. Stellenbesetzung: Patient ${ }^{*}$ innen-Pflegepersonal-Relation im internationalen Vergleich; eigene Darstellung; mod. nach [51]

\begin{tabular}{lccl}
\hline Land & $\begin{array}{l}\text { Patient*innen- } \\
\text { zu-Pflegefach- } \\
\text { kräfte-Relation }\end{array}$ & $\begin{array}{l}\text { Patient*innen- } \\
\text { zu-Pflegegesamt- } \\
\text { personal }{ }^{*} \text {-Relation }\end{array}$ & $\begin{array}{l}\text { Teilnehmende } \\
\text { Krankenhäuser } \\
(\mathrm{n})\end{array}$ \\
\hline USA & 5,3 & 3,6 & 617 \\
Norwegen & 5,4 & 3,3 & 35 \\
Irland & 6,9 & 5,0 & 30 \\
Niederlande & 7,0 & 5,0 & 28 \\
Schweden & 7,7 & 4,2 & 79 \\
Schweiz & 7,9 & 5,0 & 35 \\
Finnland & 8,3 & 5,3 & 32 \\
UK & 8,6 & 4,8 & 46 \\
Griechenland & 10,2 & 6,2 & 24 \\
Polen & 10,5 & 6,8 & 33 \\
Belgien & 10,7 & 7,9 & 67 \\
Spanien & 12,6 & 6,8 & 33 \\
Deutschland & 13,0 & 10,5 & 49 \\
\hline
\end{tabular}

*Das Pflegegesamtpersonal umfasst sowohl Pflegefachkräfte als auch geringer qualifiziertes Pflegepersonal.

Welche Bereiche sind "pflegesensitiv»?

Zur Verbesserung der Personalsituation hat die Politik inzwischen mit der Einführung von Pflegepersonaluntergrenzen (PpUGV) sowie dem Pflegepersonal-Stärkungsgesetz (PpSG) reagiert [54]. Allerdings sah der «Referentenentwurf einer Verordnung zur Festlegung von Pflegepersonaluntergrenzen in pflegesensitiven Krankenhausbereichen für das Jahr 2019» keine Berücksichtigung der Versorgung von Patient ${ }^{\star}$ innen mit hämatologischen und onkologischen Erkrankungen als «pflegesensitiven Bereich» vor. Die DGHO hat daraufhin in einer Stellungnahme klargestellt, dass der Aufwand bei der Versorgung von hämatologischen und onkologischen Patient*innen hoch ist [55]. Dazu wurde auf den stationären Versorgungbedarf bei intensiver Systemtherapie, akuten Komplikationen, Stammzelltransplantationen sowie hoher Komorbidität hingewiesen und die Untergrenzen für die pflegerische Betreuung aufgeführt, die derzeit in den onkologischen Zentren im Bereich der Stammzelltransplantation angesetzt werden (Tab. 5).
Table 5. PpUGV: Verbindliche Pflegepersonaluntergrenzen-Verordnung

Verbindliche Pflegepersonaluntergrenzen für pflegesensitive Krankenhausbereiche

Seit 2019: im Bereich Intensivmedizin/Geriatrie/Unfallchirurgie/ Kardiologie

Seit 2020: im Bereich Neurologie/Herzchirurgie/Pädiatrie

Seit Februar 2021: Innere Medizin (10:1; nachts 22:1)

Stellungnahme DGHO: Die Versorgung von Patient*innen mit hämatologischen und onkologischen Erkrankungen ist ebenfalls ein pflegesensitiver Bereich!

Gemäß DGHO an den onkologischen Zentren angesetzt (Stand der Zertifizierung: Mai 2021):

Allogene Stammzelltransplantation: 1:3; nachts 1:6 Autologe Stammzelltransplantation/Leukämie-Station: 1:4 (-5); nachts 1:8 (-10)

Onkologische Station: 1:6 (-8); nachts 1:12 (-16)

\section{Verbesserte Rahmenbedingungen gefragt}

Auch wenn die monatlichen Bruttoentgelte der Pflegebranche teilweise noch immer unter dem Durchschnittsgehalt aller Beschäftigten liegen, sind die Gehälter in den vergangenen Jahren gestiegen und Fachkräfte der Krankenpflege verdienen sogar geringfügig mehr [56]. Über die reine Entgeltsituation für Fachpflegekräfte hinaus stehen im stationären Sektor vor allem die Verbesserungen der Rahmenbedingungen im Vordergrund: Dazu gehört die für die Zukunft wichtige Akademisierung der Pflege mit dem Ausbau wissenschaftlicher Kompetenz ebenso wie die Steigerung der beruflichen Attraktivität und (im)materiellen Anerkennung. Perspektivisch bedarf es auch der stärkeren Einbeziehung von Pflegefachpersonen in die Entscheidungsgremien und mehr Mitteln für die pflegewissenschaftliche Versorgungsforschung. Gute Pflege «kostet» folglich den Einsatz aller professionell Pflegenden, Akteur*innen und Vertreter ${ }^{\star}$ innen der Pflege, Pflegewissenschaft, Politik und Ärzteschaft sowie der Kosten- und Einrichtungsträger, um die notwendigen Veränderungen gemeinsam voranzubringen.

\begin{abstract}
Literatur
1 Verordnung über die Nutzenbewertung von Arzneimitteln nach $\$ 35 \mathrm{a}$ Absatz 1 SGB V für Erstattungsvereinbarungen nach $₫ 130 \mathrm{~b}$ SGB V. https:// www.gesetze-im-internet.de/am-nutzenv/

2 Gesundheitspolitische Schriftenreihe der DGHO. (Band 2): Nutzenbewertung von Arzneimitteln der Onkologie und Hämatologie 2013. Eine Standortbestimmung. https://www.dgho. de/publikationen/schriftenreihen/fruehe-nutzenbewertung, August 2013.
\end{abstract}

3 AWMF (Arbeitsgemeinschaft der Wissenschaftlichen Medizinischen Fachgesellschaften) e. V. und DGHO (Deutsche Gesellschaft für Hämatologie und Medizinische Onkologie e.V.) (Hrsg): Frühe Nutzenbewertung neuer Arzneimittel in Deutschland (2011-2018). https:// www.dgho.de/publikationen/schriftenreihen/ fruehe-nutzenbewertung, Mai 2019.
4 Gesundheitspolitische Schriftenreihe der DGHO. (Band 12): Frühe Nutzenbewertung neuer Arzneimittel in Deutschland 2011-2017. Status und Tendenzen. https://www.dgho.de/ publikationen/schriftenreihen/fruehe-nutzenbewertung, April 2018.

5 Krebs gesamt - C00-C97 ohne C4; in Robert-Koch Institut(Hrsg): Krebsin Deutschland für 2015/2016. https://www.krebsdaten.de/Krebs/DE/Home/ homepage_node.html 
6 Bokemeyer C, Hallek M, Lüftner D, Weißinger F. Frühe Nutzenbewertung neuer Arzneimittel in Deutschland: Subgruppen. Gesundheitspolitische Schriftenreihe der DGHO (Band 8). https:/www. dgho.de/publikationen/schriftenreihen/fruehenutzenbewertung, April 2016.

7 Gesetz zur Neuordnung des Arzneimittelmarktes in der gesetzlichen Krankenversicherung (Arzneimittelmarktneuordnungsgesetz - AMNOG), Bundesgesetzblatt Teil I vom 27.12.2010. https:// www.bgbl.de/xaver/bgbl/start.xav?start= $\% 2 \mathrm{~F} \% 2 \mathrm{~F}^{*}[\% 40 \mathrm{attr}$ id\%3D\%27bgbl110s2262. pdf\%27]\#__bgbl__\%2F\%2F*\%5B\%40attr id \% 3 D \% 27bgbl110s2262.pdf\%27\%5D 1620123389798

8 Positionspapier «Frühe Nutzenbewertung neuer Arzneimittel 2019: Gerecht und nachhaltig?» https://www.awmf.org/medizin-versorgung/nutzenbewertung.html

9 Bundesausschuss G. (G-BA): Tragende Gründe zum Beschluss des Gemeinsamen Bundesausschusses über eine Änderung der Verfahrensordnung (VerfO): Änderung des 5. Kapitels vom 20. Februar 2020. https://www.g-ba.de/downloads/40-268-6375/2020-02-20_VerfO_Kapitel-5_ TrG.pdf

10 EFPIA Patients W.A.I.T. Indicator 2019 Survey (Mai 2020). https://www.efpia.eu/publications/ downloads/efpia/efpia-patients-wait-indicator2019-survey/

11 Storm A, editor. AMNOG-Report 2020: 10 Jahre AMNOG - Rückblick und Ausblick. Beiträge zur Gesundheitsökonomie und Versorgungsforschung (Band 32). https://www.dak.de/dak/download/report-2335144.pdf, September 2020.

12 Schwabe U, Ludwig WD, editors. Arzneiverordnungs-Report 2020. Berlin: Springer-Verlag; 2020 https://doi.org/10.1007/978-3-662-62168-4.

13 Bundesministerium für Gesundheit. Gesetz für mehr Sicherheit in der Arzneimittelversorgung (GSAV). Bundesgesetzblatt Teil INr.30 (15. August 2019). https://www.bundesgesundheitsministerium.de/fileadmin/Dateien/3_Downloads/Gesetze_ und_Verordnungen/GuV/G/GSAV_bgbl119_ S.1202_150819.pdf, 2019.

14 Bundesausschuss G. G-BA konkretisiert Verfahren $\mathrm{zu}$ anwendungsbegleitender Datenerhebung Gentherapie Zolgensma erster Fall. Pressemitteilung Nr. 30/2020, 16.07.2020. https://www.gba.de/presse/pressemitteilungen-meldungen/874/

15 Die forschenden Pharma-Unternehmen (vfa): Einsparungen addieren sich auf 14 Milliarden Euro. https://www.vfa.de/de/wirtschaft-politik/politik/ amnog-einsparungen, 3. Mai 2021.

16 Die forschenden Pharma-Unternehmen (vfa) Deutschland: Weltweite Nr. 5 bei klinischen Studien der Industrie. https://www.vfa.de/de/arzneimittel-forschung/forschungsstandort-deutschland/ klinische-studien-deutschland.html

17 Gesundheitspolitische Schriftenreihe der DGHO (Band 14): Deutschlandweite Prognose der bevölkerungsbezogenen Morbiditätserwartung für häufige Krebserkrankungen. Auswirkungen auf die Versorgung. https://www.dgho.de/publikationen/ schriftenreihen/demografischer-wandel/dgho gpsr_xiv_web.pdf, März 2020 (Version 2).

18 Wissenschaftliches Institut der Niedergelassenen Hämatologen und Onkologen (WINHO). Qualitätsbericht der hämatologischen und onkologischen Schwerpunktpraxen (2020). www.winho.de

19 Hermes-Moll K, Heidt V: Wandel der ambulanten onkologischen Versorgung und die Folgen. InFo
Hämatologie + Onkologie 2019;22:68-71. https:// doi.org/10.1007/s15004-019-6582-1.

20 Heidt V, Knauf W, Illmer T, Engel E, Goetzenich A. Trotz Pandemie ambulant gut versorgt. Dtsch Arztebl. 2021;118:A310-3.

21 Zentralinstitut für kassenärztliche Versorgung (Zi): Preisentwicklung für ärztliche Leistungen auf niedrigem Niveau. https://www.zi.de/presse/archiv/ grafik-des-monats/2018, August 2018.

22 Graf von Stillfried D. Ambulante Versorgungsleistungen. In: Haring R, editor. Gesundheitswissenschaften. Berlin, Heidelberg: Springer; 2019. https:// doi.org/10.1007/978-3-662-58314-2_52.

23 Zentralinstitut für kassenärztliche Versorgung (Zi): Zi-Praxis-Panel - Zentrale Ergebnisse aus der Befragung 2019 zu den Berichtsjahren 2015 bis 2018. Berlin, Oktober 2020 (Vorabinformation).

24 Zentralinstitut für kassenärztliche Versorgung (Zi): Zi-Erhebung «Besondere Kosten im Praxismanagement». Berlin, 2020.

25 Statistisches Bundesamt. (Destatis), https://www. destatis.de/DE/Presse/Pressemitteilungen/2021/02/PD21_N010_231.html, 2021.

26 Kassenärztliche Bundesvereinigung (KBV). Medizinische Versorgungszentren. Statistische Informationen zum Stichtag 21.12.2019. https://www. kbv.de/media/sp/mvz-aktuell.pdf

27 Kassenärztliche Bundesvereinigung (KBV). Berufsmonitoring Medizinstudierende 2018. Ergebnisse einer bundesweiten Befragung. Berlin, 2019.

28 Baumann W, Schmitz S. Entwicklungen in der onkologischen Versorgung. Onkologe. 2016;22(3):158-66.

29 Coenen M, Haucap J, Hottenrott M. Wettbewerb in der ambulanten onkologischen Versorgung: Analyse und Reformansätze, DICE. Ordnungspolitische Perspektiven, No. 81. Düsseldorf, Düsseldorf Institute for Competition Economics (DICE), 2016, ISBN 978-3-86304-681-1.

30 Brandhorst A, Hildebrandt H. Kooperation und Integration - das unvollendete Projekt des Gesundheitswesens: Wie kommen wir weiter? In: Brandhorst A, Hildebrandt H, Luthe EW, editors. Kooperation und Integration - das unvollendete Projekt des Gesundheitssystems. Politik - Gesellschaft Wirtschaft. Wiesbaden: Springer VS; 2017. https:// doi.org/10.1007/978-3-658-13783-0_31.

31 Antrag derFraktion BÜNDNID 90/DIEGRÜNEN: Gesundheitsregionen - Aufbruch für mehr Verlässlichkeit, Kooperation und regionale Verankerung in unserer Gesundheitsversorgung. Bundestagsdrucksache 19/21881 vom 26.08.2020.

32 Unterrichtung des deutschen Ethikrates: Stellungnahme des nationalen Ethikrates - Patientenwohl als ethischer Maßstab für das Krankenhaus. Bundestagsdrucksache 18/8843 vom 07.06.2016.

33 Jörg J. Berufsethos kontra Ökonomie: Haben wir in der Medizin zu viel Ökonomie und zu wenig Ethik? Berlin, Heidelberg: Springer-Verlag; 2015. https:// doi.org/10.1007/978-3-662-47066-4.

34 Aufgedeckt: Kliniken und Bilanzdruck: Befehl von oben. DAZ.online vom 30.03.2016.

35 Wehkamp KH, Naegler $\mathrm{H}$. The commercialization of patient-related decision-making in hospitals - a qualitative study of the perceptions of doctors and chief executive officers. Dtsch Arztebl Int. 2017 Nov;114(47):797-804.

36 Schönmayr R. Ethik und Ökonomie in der Medizin. Unversöhnliche Feinde. Rotary Magazin. 2013;(1):54-7.

37 Köbberling J. Economic pressure in hospitals - profound disagreement over priorities between doctors and managers. Dtsch Arztebl Int. 2017 Nov;114(47):795-6.

38 Richter W: Medizin-Controller und Chefarzt - ein starkes Team? Controlling Management Review 2015, Sonderheft 3.

39 Klauber J, Geraedts M, Friedrich J, Wasem J, Beivers A, editors. Krankenhausreport 2020. Berlin: Springer; 2020. https://doi.org/10.1007/978-3-662-604878.

40 Bode I, Vogd W, editors. Mutationen des Krankenhauses. Wiesbaden: Springer Fachmedien; 2016. https://doi.org/10.1007/978-3-658-11853-2.

41 Scheuplein C, Evans M, Merkel S: Übernahmen durch Private Equity im deutschen Gesundheitssektor. An Assessment for the Years 2013 to 2018. IAT Discussion Papers 2019, 19/1, January.

42 Kowalski C, Albert US, Baumann W, Carl EG, Ernstmann N, Hermes-Moll K, et al. DNVF-Memorandum Versorgungsforschung in der Onkologie. Gesundheitswesen. 2020 Sep;82(8-09):e108-21.

43 Deutsche Krebsgesellschaft (DKG). Jahresbericht 2020 der zertifizierten Onkologischen Zentren. www.krebsgesellschaft.de

44 Prognos 2018: Endbericht «Nutzen, Mehraufwand und Finanzierung von Onkologischen Spitzenzentren, Onkologischen Zentren und Organkrebszentren». Im Auftrag der Deutschen Krebshilfe und der Deutschen Krebsgesellschaft.

45 Klein F. Spitzenversorgung braucht stabile Finanzierung. Im Fokus Onkologie 2018;21:70-71. https://doi.org/10.1007/s15015-018-4303-5.

46 Beruf M. Pflege - Das Manifest der Pflegeberufe. https://www.dbfk.de/manifest/der-hintergrund/, 2015.

47 Fricke A: Onkologische Pflege - Es fehlt an 70.000 Pflegekräften. ÄrzteZeitung vom 22.08.2018.

48 Zander B, Dobler L, Bäumler M, Busse R. Implizite Rationierung von Pflegeleistungen in deutschen Akutkrankenhäusern - Ergebnisse der internationalen Pflegestudie RN4Cast. Gesundheitswesen. 2014 Nov;76(11):727-34.

49 Pflegedebatte beim DRG-Forum: Es braucht einen kräftigen Ruck. DRG-Forum News vom 19.03.2021.

50 DGF und DIVI: Stellungnahme zur Stärkung und Zukunft der Intensivpflege in Deutschland. https:// www.divi.de, 2021.

51 Aiken LH, Sloane DM, Bruyneel L, Van den Heede K, Sermeus W; RN4CAST Consortium. Nurses' reports of working conditions and hospital quality of care in 12 countries in Europe. Int J Nurs Stud. 2013 Feb;50(2):143-53.

52 Aiken LH, Sloane DM, Bruyneel L, Van den Heede K, Griffiths P, Busse R, et al.; RN4CAST consortium. Nurse staffing and education and hospital mortality in nine European countries: a retrospective observational study. Lancet. 2014 May;383(9931):1824-30.

53 Wissenschaftsrat: Empfehlungen zu hochschulischen Qualifikationen für das Gesundheitswesen. Drucksache 2411-12, Berlin, 2012.

54 Gesetz zur Stärkung des Pflegepersonals (Pflegepersonal-Stärkungsgesetz - PpSG) vom 11. Dezember 2018. Bundesgesetzblatt Jahrgang 2018 Teil I Nr. 45, ausgegeben zu Bonn am 14.12.2018.

55 DGHO: Stellungnahme zum Referentenentwurf einer Verordnung zur Festlegung von Pflegepersonaluntergrenzen in pflegesensitiven Krankenhausbereichen für das Jahr 2019 (Pflegepersonaluntergrenzen-Verordnung - PpUGV) vom 13.09.2018.

56 Seibert H, Carstensen J, Wiethölter D. Entgelte von Pflegekräften - weiterhin große Unterschiede zwischen Berufen. IAB-Forum, 16.01.2018. 